\title{
REFLECTION AND TRANSMISSION AT THE BOUNDARY SURFACE OF MODIFIED COUPLE STRESS THERMOELASTIC MEDIA
}

\author{
R. KUMAR \\ Department of Mathematics, Kurukshetra University \\ Kurukshetra, Haryana, INDIA-136119 \\ E-mail: Rajneesh_kuk@rediffmail.com \\ K. KUMAR* \\ N.C. College of Engineering \\ Israna, Panipat, Haryana, INDIA-132107 \\ E-mail: dahiya_krishan@rediffmail.com
}

\begin{abstract}
In this paper the reflection and transmission at a plane interface in modified couple stress generalized thermoelastic solid half spaces in the context of Loard-Shulman (LS) and Green-Lindsay (GL) theories in welded contact are investigated. Amplitude ratios of various reflected and transmitted waves are obtained due to incidence of a set of coupled longitudinal waves and coupled transverse waves. It is found that the amplitude ratios of various reflected and transmitted waves are functions of the angle of incidence, frequency and are affected by the couple stress properties of the media. Some special cases are deduced from the present formulation.
\end{abstract}

Key words: modified couple stress thermoelastic solid, longitudinal wave, transverse wave, reflection, transmission, amplitude ratios.

Classifications: 74B05, 74F05, 74J15.

\section{Introduction}

The classical couple stress theory was developed by Cosserts [1], Toupin [2] and Mindlin and Tiersten [3], which is one class of the higher-order continuum theories, contains two classical and two additional material constants for isotropic elastic materials [4]. The two additional constants are related to the underlying microstructure of the material and are inherently difficult to determine. Yang et al. [5] first proposed the modified couple stress theory by introducing the concept of the representative element and defining the force and couple applied to a single material particle. In this theory, the constitutive equations involved only one additional material length scale parameter besides two classical material constants. After Yang et al.'s [5] pioneering work, the modified couple stress theory has been concentrated on and developed in the past several years. Gao and Reddy [7] discussed a microstructure-dependent Timoshenko beam model based on a modified couple stress theory. Park and Gao [8] presented micromechanical modeling of honeycomb structures based on a modified couple stress theory. Yin et al. [6] investigated vibration analysis of microscale plates based on modified couple stress theory. Şimşek [9] investigated dynamic analysis of an embedded microbeam carrying a moving microparticle based on the modified couple stress theory. Jomehzadeh et al. [10] investigated size-dependent vibration analysis of micro-plates based on a modified couple stress theory. Gao and Reddy [11] presented a non-classical Mindlin plate model based on a modified

\footnotetext{
* To whom correspondence should be addressed
} 
couple stress theory. Akgöz and Civalek [12] discussed free vibration analysis for single-layered graphene sheets in an elastic matrix via modified couple stress theory.

Nateghi and Salamat-Talab [13] studied the thermal effect on the size dependent behavior of functionally graded microbeams based on modified couple stress theory. A new modified couple stress theory for anisotropic elasticity and microscale laminated Kirchhoff plate model was studied by Chen and Li [14]. Gao et al. [15] presented a non-classical third-order shear deformation plate model based on a modified couple stress theory. Ansari et al. [16] studied the nonlinear vibrations of functionally graded Mindlin microplates based on the modified couple stress theory. Kahrobaiyan et al. [17] presented a Timoshenko beam element based on the modified couple stress theory. Abadi and Daneshmehr [18; 19] presented an investigation of modified couple stress theory in buckling analysis of micro composite laminated EulerBernoulli and Timoshenko beams and size dependent buckling analysis of microbeams based on modified couple stress theory with high order theories and general boundary conditions.

Morini et al. [20] presented remarks on the energy release rate for an antiplane moving crack in couple stress elasticity. Şimşek [21] investigated the nonlinear static and free vibration analysis of microbeams based on the nonlinear elastic foundation using the modified couple stress theory. Ashoori and Mahmoodi [22] derived the modified version of strain gradient and couple stress theories in general curvilinear coordinates. Askari and Tahani [23] investigated size-dependent dynamic pull-in analysis of beam-type MEMS under mechanical shock based on the modified couple stress theory. Dehrouyeh-Semnani and Nikkhah-Bahrami [24] presented a discussion on incorporating the Poisson effect in microbeam models based on the modified couple stress theory. Farokhi and Ghayesh [25] investigated nonlinear dynamical behaviour of geometrically imperfect microplates based on the modified couple stress theory. Beni, Mehralian and Razavi [26] carried out free vibration analysis of size-dependent shear deformable functionally graded cylindrical shell on the basis of the modified couple stress theory.

Biot [27] formulated the theory of coupled thermoelasticity to eliminate the paradox inherent in the classical uncoupled theory that elastic changes have no effect on the temperature. The heat equations for both the theories are of parabolic type predicting infinite speeds of propagation for heat waves contrary to physical observations. Lord and Shulman [28] introduced relaxation time to explain the propagation of a thermal wave and presented a modified theory called the generalized theory of thermoleasticity (termed as LS theory). Using two relaxation times, Green and Lindsay [29] developed another generalized theory of thermoelasticity (termed as GL theory). Ram and Sharma [30] discussed reflection and transmission of micropolar thermoelastic waves with an imperfect bonding. Xue et al. [31] studied reflection and refraction of longitudinal displacement wave at an interface between two micropolar elastic solids. Kumar et al. [32] presented reflection and transmission between two micropolar thermoelastic half-spaces with three-phase-lag model. Reflection at the free surface of a couple stress generalized thermoelstic solid half-space was investigated by Kumar et al. [33].

In the present paper, the reflection and transmission phenomenon at a plane interface in the modified couple stress theory has been analyzed. In a modified couple stress thermoelastic solid medium, potential functions are introduced to represent two longitudinal waves and two transverse waves. The amplitude ratios of various reflected and transmitted waves to that of the incident wave are derived numerically and depicted graphically.

\section{Basic equations}

Following Yang et al. [5], Lord-Shulman [28] and Green-Lindsay [29] the governing equations in a couple-stress generalized thermoelastic solid are given by

$$
\begin{aligned}
& t_{j i, j}=\rho \ddot{u}_{i}, \\
& m_{j i, j}+e_{i j k} t_{j k}=0,
\end{aligned}
$$




$$
\begin{aligned}
& K^{*} \nabla^{2} T-\rho c_{e}\left(\frac{\partial T}{\partial t}+\tau_{0} \frac{\partial^{2} T}{\partial t^{2}}\right)=T_{o} \beta\left(\frac{\partial u_{i, i}}{\partial t}+\tau_{0} n_{o} \frac{\partial^{2} u_{i, i}}{\partial t^{2}}\right), \\
& t_{i j}=\lambda \varepsilon_{k k} \delta_{i j}+2 \mu \varepsilon_{i j}-\frac{1}{2} e_{i j k} m_{l k, l}-\beta\left(1+\tau_{1} \frac{\partial}{\partial t}\right) T \delta_{i j}, \\
& m_{i j}=2 \mu l^{2} \phi_{i, j}, \\
& k_{i j}=\frac{1}{2}\left(\phi_{i, j}+\phi_{j, i}\right), \\
& \phi_{i}=\frac{1}{2} e_{i p q} u_{q, p}
\end{aligned}
$$

where $t_{i j}$ is the stress components, $\varepsilon_{i j}$ is the strain components, $u_{i}$ is the displacement components, $m_{i j}$ is the couple-stress components, $\delta_{i j}$ is Kronecker's delta, $e_{i j k}$ is the alternate tensor, $k_{i j}$ is the curvature tensor, $\rho$ is the density, $\phi_{i}$ is the rotational vector, $\tau_{0}, \tau_{1}$ are thermal relaxation times with $\tau_{l} \geq \tau_{0} \geq 0$. Here $n_{o}=1, \tau_{1}=0$, for L-S theory and $n_{o}=0$ for G-L theory.

Equation (2.1) with the help of Eqs (2.2), (2.4)-(2.7) takes the form

$$
(\lambda+\mu) u_{j, i j}+\mu \nabla^{2} u_{i}+\frac{\mu l^{2}}{4}\left(e_{i j k} e_{k p q} u_{q, p i}\right), l l-\beta\left(1+\tau_{1} \frac{\partial}{\partial t}\right) T,_{i}=\rho \ddot{u}_{i} .
$$

\section{Formulation of the problem}

We consider two modified couple stress homogenous isotropic generalized thermoelastic solid half spaces medium $M_{1}\left(0<x_{3}<\infty\right)$ and medium $M_{2}\left(-\infty<x_{3}<0\right)$. The two media are in contact with each other at the plane $x_{3}=0$ of the rectangular Cartesian coordinate system $\left(x_{1}, x_{2}, x_{3}\right)$ with an origin on the surface $x_{3}=0$ and $x_{3}$ axis pointing vertically downwards into the medium $M_{1}$. We choose the $x_{1}$ axis in the direction of wave propagation so that all particles on the line parallel to $x_{2}$ axis are equally displaced. Therefore all the field quantities are independent of $x_{2}$. For two dimensional problems, we assume the displacement vector as

$$
u_{i}=\left(u_{1}, 0, u_{3}\right)
$$

We introduce the potential functions $\phi$ and $\psi$ through the relation

$$
u_{1}=\frac{\partial \phi}{\partial x_{1}}-\frac{\partial \psi}{\partial x_{3}}, \quad u_{3}=\frac{\partial \phi}{\partial x_{3}}+\frac{\partial \psi}{\partial x_{1}}
$$

We define the dimensionless quantities as 


$$
\begin{aligned}
& x_{1}^{\prime}=\frac{\omega^{*}}{c_{1}} x_{1}, \quad x_{3}^{\prime}=\frac{\omega^{*}}{c_{1}} x_{3}, \quad t^{\prime}=\omega^{*} t, \quad t_{i j}^{\prime}=\frac{t_{i j}}{\beta T_{0}}, \quad m_{i j}^{\prime}=\frac{\omega^{*} m_{i j}}{c_{l} \beta T_{0}}, \\
& c_{1}^{2}=\frac{\lambda+2 \mu}{\rho}, \quad \omega^{* 2}=\frac{4 \lambda^{2}}{\rho \mu l^{2}}, \quad u_{1}^{\prime}=\frac{\omega^{*}}{c_{1}} u_{1}, \quad u_{3}^{\prime}=\frac{\omega^{*}}{c_{1}} u_{3}, \quad T^{\prime}=\frac{T}{T_{0}}, \\
& \tau_{1}^{\prime}=\omega^{*} \tau_{1}, \quad \tau_{0}^{\prime}=\omega^{*} \tau_{0}, \quad t_{0}^{\prime}=\omega^{*} t_{0}, \quad h^{\prime}=\frac{c_{l} h}{\omega^{*}} .
\end{aligned}
$$

Upon introducing the quantities defined by Eqs (3.3) in Eq.(2.8) and with the aid of Eqs (2.4)-(2.7), (3.1) and (3.2) after suppressing the primes, we obtain

$$
\begin{aligned}
& \nabla^{2} \phi-a_{13}\left(1+\tau_{1} \frac{\partial}{\partial t}\right) T=\ddot{\phi}, \\
& \nabla^{2} \psi-a_{12} \nabla^{4} \psi=\frac{c_{1}^{2}}{c_{2}^{2}} \ddot{\psi}, \\
& \nabla^{2} T-a_{6}\left(\frac{\partial T}{\partial t}+\tau_{0} \frac{\partial^{2} T}{\partial t^{2}}\right)=a_{7} \delta_{i j}\left(\frac{\partial u_{i, j}}{\partial t}+\tau_{0} n_{o} \frac{\partial^{2} u_{i, j}}{\partial t^{2}}\right), \\
& t_{i j}=a_{1} \delta_{i j} e+a_{2}\left(u_{i, j}+u_{j, i}\right)-a_{3} e_{i j k} e_{k p q} u_{q, p l l}-\beta\left(1+\tau_{1} \frac{\partial}{\partial t}\right) T \delta_{i j}, \\
& m_{i j}=\frac{1}{2}\left(a_{4} e_{i p q} u_{q, p j}+a_{4} e_{j p q} u_{q, p i}\right)
\end{aligned}
$$

where

$$
\begin{aligned}
& a_{1}=\frac{\lambda}{\beta T_{0}}, \quad a_{2}=\frac{\mu}{\beta T_{0}}, \quad a_{3}=\frac{\mu l^{2} \omega^{*^{2}}}{4 c_{1}^{2} \beta T_{0}}, \quad a_{4}=\frac{\mu l^{2} \omega^{* 2}}{2 c_{1}^{2} \beta T_{0}}, \quad a_{6}=\frac{\rho c_{e} c_{1}^{2}}{K^{*} \omega^{*}}, \\
& a_{7}=\frac{c_{1}^{2} \beta}{K^{*} \omega^{*}}, \quad a_{8}=\frac{\mu}{\lambda+\mu}, \quad a_{9}=\frac{\mu l^{2} \omega^{* 2}}{4 c_{1}^{2}(\lambda+\mu)}, \quad a_{10}=\frac{\beta T_{0}}{\lambda+\mu}, \\
& a_{11}=\frac{\rho c_{1}^{2}}{\lambda+\mu}, \quad a_{12}=\frac{a_{9}}{a_{8}}, \quad a_{13}=\frac{a_{10}}{1+a_{8}} .
\end{aligned}
$$

Equation (3.5) corresponds to a purely transverse wave mode that decouples from rest of the motion and is not affected by the thermal effect but affected by the effect of couple stress.

We assume the solution of the form

$$
(\phi, \psi, T)=\left(\phi_{1}, \psi_{1}, T_{1}\right) e^{\left\{i\left(k\left(x_{1} \sin \theta-x_{3} \cos \theta\right)-\omega t\right)\right\}}
$$


where $k$ is the wave number and $\omega$ is the circular frequency.

Substituting the values of $\phi, \psi$ and $T$ from Eq.(3.9) in Eqs (3.4)-(3.6), we obtain

$$
\begin{aligned}
& A V^{4}+B V^{2}+C=0 \\
& D V^{4}+E V^{2}+F=0
\end{aligned}
$$

where $V=\frac{\omega}{k}$ is the velocity of waves, and

$$
A=l_{1} a_{7}, \quad B=-a_{7} l_{1}-1-i \omega a_{4} a_{8} l_{2} l_{3}, \quad C=1, \quad D=\frac{c_{1}^{2}}{a_{6} c_{2}^{2}}, \quad E=-\frac{1}{a_{6}}, \quad F=-\omega^{2} .
$$

Let the roots of Eq.(3.10) be denoted by $V_{i}^{2}(i=1,2)$. Two positive values of $V$ in the descending order will be the velocities of longitudinal waves (P) and thermal waves (T). Similarly, the roots of Eq.(3.11) will be denoted by $V_{i}^{2}(i=3,4)$ and two positive roots of $V$ of Eq.(3.11) are the velocities of reflected transverse waves (SV1 and SV2).

\section{Reflection and transmission}

We consider longitudinal waves $(\mathrm{P})$ or thermal waves $(\mathrm{T})$ or transverse waves (SV1 or SV2) propagating through the medium $M_{1}$ and incident at the plane $x_{3}=0$. Corresponding to each incident wave, we get reflected longitudinal wave (P), thermal wave (T), transverse waves (SV1 and SV2) in medium $M_{1}$ and transmitted longitudinal wave(P), thermal wave (T), transverse waves (SV1 and SV2) in medium $M_{2}$ as shown in Fig.1.

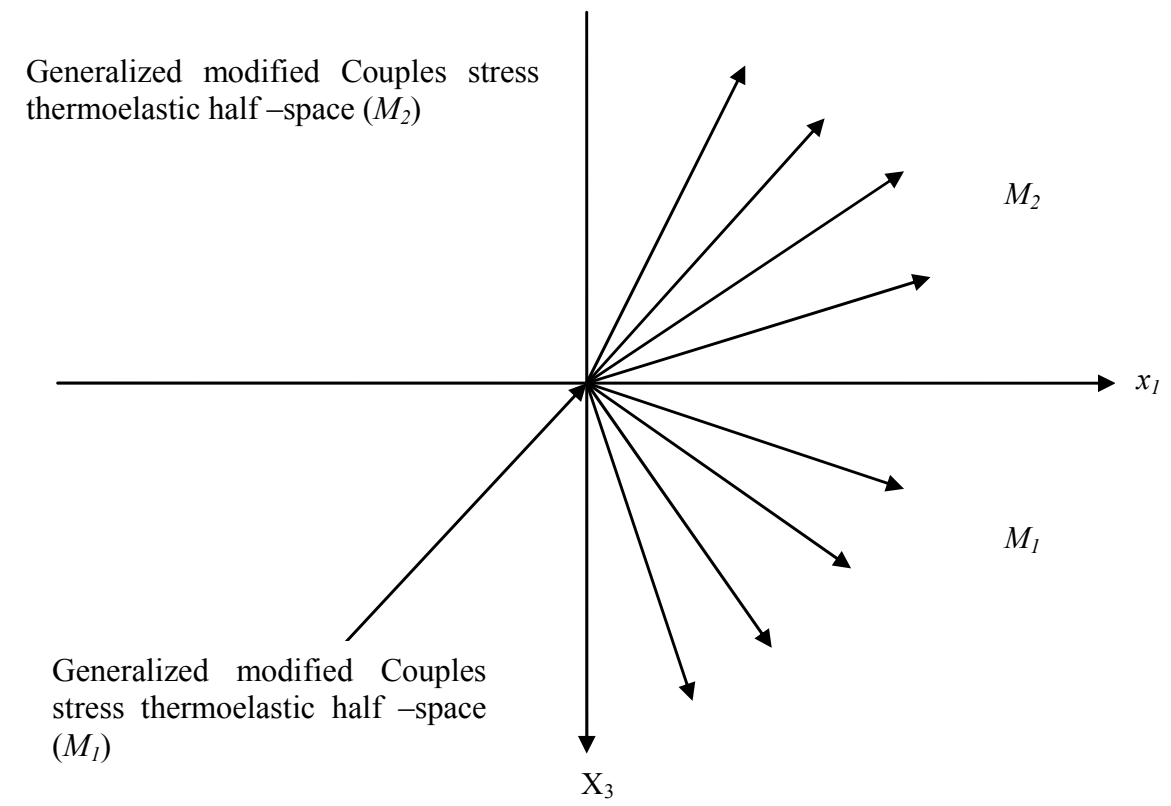

Fig.1. 


\section{Boundary conditions}

\section{Mechanical Conditions}

The boundary conditions are the continuity of normal stress, tangential stress, tangential couple stress, displacement components and rotations vector at the interface of two modified couple stress generalized thermoelastic solid half-spaces. Mathematically, these can be written as

$$
\left.\begin{array}{l}
t_{33}=\bar{t}_{33} \\
t_{31}=\bar{t}_{31} \\
m_{32}=\bar{m}_{32} \\
u_{1}=\bar{u}_{1} \\
u_{3}=\bar{u}_{3} \\
\phi_{2}=\bar{\phi}_{2}
\end{array}\right\} \text { at } x_{3}=0 .
$$

Thermal Condition: The thermal condition corresponding to the insulated boundary is given by

$$
\left.\begin{array}{c}
T=\bar{T} \\
K * \frac{\partial T}{\partial x_{3}}=\bar{K} * \frac{\partial \bar{T}}{\partial x_{3}}
\end{array}\right\} \quad \text { at } \quad x_{3}=0
$$

In view of Eq.(3.9) we assume the values of $\phi, \psi$ and $T$ for medium $M_{1}$ as

$$
\begin{aligned}
& (\phi, T)=\sum_{i=1}^{2}\left(1, b_{i}\right)\left[A_{0 i} e^{\left\{i\left(k\left(x_{1} \sin \theta_{0 i}-x_{3} \cos \theta_{0 i}\right)-\omega t\right)\right\}}+P_{i}\right], \\
& \psi=\sum_{j=3}^{4} B_{0 j}\left[e^{\left\{i\left(k\left(x_{l} \sin \theta_{0 j}-x_{3} \cos \theta_{0 j}\right)-\omega t\right)\right\}}+P_{j}\right] .
\end{aligned}
$$

We attach bar notation for medium $M_{2}$, then the values of $\bar{\varphi}, \bar{\psi}$ and $\bar{T}$ are taken as

$$
\begin{aligned}
& (\bar{\phi}, \bar{T})=\sum_{i=1}^{2}\left(1, \bar{b}_{i}\right)\left[\bar{A}_{i} e^{\left\{i\left(k\left(x_{I} \sin \bar{\theta}_{i}+x_{3} \cos \bar{\theta}_{i}\right)-\omega t\right)\right\}}\right], \\
& \bar{\psi}=\sum_{j=3}^{4}\left[\bar{B}_{j} e^{\left\{i\left(k\left(x_{l} \sin \bar{\theta}_{j}+x_{3} \cos \bar{\theta}_{j}\right)-\omega t\right)\right\}}\right]
\end{aligned}
$$

where

$$
P_{i}=A_{i} e^{\left\{i\left(k\left(x_{I} \sin \theta_{I}+x_{3} \cos \theta_{i}\right)-\omega t\right)\right\}}, \quad P_{j}=e^{\left\{i\left(k\left(x_{I} \sin \theta_{j}+x_{3} \cos \theta_{j}\right)-\omega t\right)\right\}},
$$




$$
b_{i}=\frac{\left(-1+V_{i}^{2}\right) k^{2}}{a_{4}\left(1-i k V_{i}^{2} \tau_{1}\right)}, \quad \bar{b}_{i}=\frac{\left(-1+\bar{V}_{i}^{2}\right) k^{2}}{\bar{a}_{4}\left(1-i k \bar{V}_{i}^{2} \bar{\tau}_{l}\right)} .
$$

$A_{0 i}$ and $A_{i}(i=1,2)$ are the amplitudes of incident and reflected longitudinal waves (P) and thermal waves (T), respectively, where $B_{0 j}$ and $B_{j}(j=3,4)$ are the amplitudes of incident and reflected transverse waves (SV1 and SV2), respectively. $\bar{A}_{i}(i=1,2)$ and $\bar{B}_{j}(j=3,4)$ are the amplitudes of transmitted plane waves (P), thermal waves (T) transmitted transverse waves (SV1 and SV2), respectively.

In order to satisfy the boundary conditions, the extension of Snell's law is given by

$$
\frac{\sin \theta_{0}}{V_{0}}=\frac{\sin \theta_{1}}{V_{1}}=\frac{\sin \theta_{2}}{V_{2}}=\frac{\sin \theta_{3}}{V_{3}}=\frac{\sin \theta_{4}}{V_{4}}=\frac{\sin \bar{\theta}_{1}}{\bar{V}_{1}}=\frac{\sin \bar{\theta}_{2}}{\bar{V}_{2}}=\frac{\sin \bar{\theta}_{3}}{\bar{V}_{3}}=\frac{\sin \bar{\theta}_{4}}{\bar{V}_{4}}
$$

where

$$
V_{1}=V_{2}=V_{3}=V_{4}=\bar{V}_{1}=\bar{V}_{2}=\bar{V}_{3}=\bar{V}_{4}=V \quad \text { at } \quad x_{3}=0 .
$$

Making use of potentials given by Eqs (5.3)-(5.6) in the boundary conditions given by Eqs (5.1) and (5.2) and with aid of Eqs (5.7) and (5.8), we obtain a system of eight non-homogeneous equations, which can be written as

$$
\sum_{i, j=1}^{8} d_{i j} Z_{j}=Y_{i}
$$

where

$$
\begin{aligned}
& d_{1 i}=-k^{2} a_{9}-k^{2} a_{10} \frac{1}{V_{0}^{2}}\left[\left(\frac{V_{0}}{V_{i}}\right)^{2}-\sin ^{2} \theta_{0}\right]-\delta_{1} b_{i}, d_{1 j}=-k^{2} a_{10} \frac{1}{V_{0}^{2}}\left[\left(\frac{V_{0}}{V_{j}}\right)^{2}-\sin ^{2} \theta_{0}\right]^{\frac{1}{2}} \sin \theta_{j}, \\
& d_{1 l}=k^{2} \bar{a}_{9}+k^{2} \bar{a}_{10} \frac{1}{V_{0}^{2}}\left[\left(\frac{V_{0}}{\bar{V}_{i}}\right)^{2}-\sin ^{2} \theta_{0}\right]+\delta_{1} \bar{b}_{i}, \quad d_{1 m}=k^{2} \bar{a}_{10} \frac{1}{V_{0}^{2}}\left[\left(\frac{V_{0}}{\bar{V}_{j}}\right)^{2}-\sin ^{2} \theta_{0}\right]^{\frac{1}{2}} \sin \bar{\theta}_{j}, \\
& d_{2 i}=-2 a_{10} k^{2} \frac{1}{V_{0}^{2}}\left[\left(\frac{V_{0}}{V_{i}}\right)^{2}-\sin ^{2} \theta_{0}\right]^{\frac{1}{2}} \sin \theta_{i}, \quad d_{2 j}=a_{10} k^{2} \frac{1}{V_{0}^{2}}\left[\left(\frac{V_{0}}{V_{j}}\right)^{2}-\sin ^{2} \theta_{0}\right]+a_{1 k^{k}}{ }^{4}, \\
& d_{2 l}=2 \bar{a}_{10} k^{2} \frac{1}{V_{0}^{2}}\left[\left(\frac{V_{0}}{\bar{V}_{i}}\right)^{2}-\sin ^{2} \theta_{0}\right]^{\frac{1}{2}} \sin \bar{\theta}_{i}, \quad d_{2 m}=-\bar{a}_{10} k^{2} \frac{1}{V_{0}^{2}}\left[\left(\frac{V_{0}}{\bar{V}_{j}}\right)^{2}-\sin ^{2} \theta_{0}\right]^{\frac{1}{2}}+a_{11} k^{4},
\end{aligned}
$$




$$
\begin{aligned}
& d_{3 j}=-a_{12} \frac{1}{V_{0}^{2}}\left[\left(\frac{V_{0}}{V_{j}}\right)^{2}-\sin ^{2} \theta_{0}\right]^{\frac{1}{2}}, \quad d_{3 m}=\bar{a}_{12} \frac{1}{V_{0}^{2}}\left[\left(\frac{V_{0}}{\bar{V}_{j}}\right)^{2}-\sin ^{2} \theta_{0}\right]^{\frac{1}{2}}, \\
& d_{4 i}=\sin \theta_{i}, \quad d_{4 j}=-a_{12} \frac{1}{V_{0}^{2}}\left[\left(\frac{V_{0}}{V_{j}}\right)^{2}-\sin ^{2} \theta_{0}\right]^{\frac{1}{2}}, \quad d_{4 l}=-\sin \bar{\theta}_{i}, \quad d_{4 m}=\bar{a}_{12} \frac{1}{V_{0}^{2}}\left[\left(\frac{V_{0}}{\bar{V}_{j}}\right)^{2}-\sin ^{2} \theta_{0}\right]^{\frac{1}{2}}, \\
& d_{5 i}=\frac{1}{V_{0}^{2}}\left[\left(\frac{V_{0}}{V_{i}}\right)^{2}-\sin ^{2} \theta_{0}\right]^{\frac{1}{2}}, \quad d_{5 j}=\sin \theta_{j}, \quad d_{5 l}=-\frac{1}{V_{0}^{2}}\left[\left(\frac{V_{0}}{\bar{V}_{i}}\right)^{2}-\sin ^{2} \theta_{0}\right]^{\frac{1}{2}}, \quad d_{5 j}=-\sin \bar{\theta}_{j}, \\
& d_{6 j}=-k^{2}, \quad d_{6 l}=k^{2}, \quad d_{7 i}=b_{i}, \quad d_{7 l}=-\bar{b}_{i}, \quad d_{8 i}=K^{*} b_{i} \frac{1}{V_{0}^{2}}\left[\left(\frac{V_{0}}{V_{i}}\right)^{2}-\sin ^{2} \theta_{0}\right]^{\frac{1}{2}}, \\
& d_{8 l}=\bar{K}^{*} \bar{b}_{i} \frac{1}{V_{0}^{2}}\left[\left(\frac{V_{0}}{\bar{V}_{i}}\right)^{2}-\sin ^{2} \theta_{0}\right]^{\frac{1}{2}}, \quad i=1,2, \quad j=3,4, \quad l=5,6, \quad m=7,8, \\
& Z_{1}=\frac{A_{1}}{A^{*}}, \quad Z_{2}=\frac{A_{2}}{A^{*}}, \quad Z_{3}=\frac{B_{1}}{A^{*}}, \quad Z_{4}=\frac{B_{2}}{A^{*}}, \quad Z_{5}=\frac{\bar{A}_{1}}{A^{*}}, \quad Z_{6}=\frac{\bar{A}_{2}}{A^{*}}, \quad Z_{7}=\frac{\bar{B}_{1}}{A^{*}}, \quad Z_{8}=\frac{\bar{B}_{2}}{A^{*}}, \\
& V_{0}=\left\{V_{1} \quad\right. \text { for incident P-wave } \\
& V_{3} \quad \text { for incident SV1-wave } \\
& V_{4} \quad \text { for incident SV2-wave\}. }
\end{aligned}
$$

and

$V_{2}$ forincident T-wave

\section{For incident P-wave}

$$
\begin{aligned}
& A^{*}=A_{01}, \quad A_{02}=B_{01}=B_{02}=0, \\
& Y_{1}=-d_{11}, \quad Y_{2}=d_{21}, \quad Y_{3}=d_{31}, \quad Y_{4}=-d_{41}, \quad Y_{5}=d_{51}, \quad Y_{6}=d_{61}, \quad Y_{7}=-d_{71}, \quad Y_{8}=d_{81} .
\end{aligned}
$$

\section{For incident T-wave}

$$
\begin{aligned}
& A^{*}=A_{02}, \quad A_{01}=B_{01}=B_{02}=0, \\
& Y_{1}=-d_{12}, \quad Y_{2}=d_{22}, \quad Y_{3}=d_{32}, \quad Y_{4}=-d_{42}, \quad Y_{5}=d_{52}, \quad Y_{6}=d_{62}, \quad Y_{7}=-d_{72}, \quad Y_{8}=d_{82} .
\end{aligned}
$$




\section{For incident SV1- wave}

$$
\begin{aligned}
& A^{*}=B_{01}, \quad A_{01}=A_{02}=B_{02}=0, \\
& Y_{1}=d_{13}, \quad Y_{2}=-d_{23}, \quad Y_{3}=d_{33}, \quad Y_{4}=d_{43}, \quad Y_{5}=-d_{53}, \quad Y_{6}=-d_{63}, \quad Y_{7}=d_{73}, \quad Y_{8}=d_{83} .
\end{aligned}
$$

\section{For incident SV2- wave}

$$
\begin{aligned}
& A^{*}=B_{02}, \quad A_{01}=A_{02}=B_{01}=0, \\
& Y_{1}=d_{14}, \quad Y_{2}=-d_{24}, \quad Y_{3}=d_{34}, \quad Y_{4}=d_{44}, \quad Y_{5}=-d_{54}, \quad Y_{6}=-d_{64}, \quad Y_{7}=d_{74}, \quad Y_{8}=d_{84} .
\end{aligned}
$$

\section{Special cases}

(i) If $n_{0}=0, \tau_{1}>0$ in Eq.(5.9) gives the resulting expression for the modified couple stress themoelastic medium with two relaxation times.

(ii) If $n_{0}=1, \tau_{1}=0$ in Eq.(5.9) gives the value of the resulting expression for the modified couple stress thermoelastic medium with one relaxation time.

(iii) If $\tau_{0}=\tau_{l}=0=n_{0}$ in Eq.(5.9) we find the resulting expression for the modified couple stress thermoelastic media.

\section{Numerical results and discussion}

We now consider a numerical example to explain the analytical procedure presented earlier for LS and GL theories with modified couple stress effect and without couple stress effect. The absolute values of amplitude ratio are computed for the angle of incidence lying between $0^{\circ} \leq \theta_{0} \leq 90^{\circ}$. Numerical computations of these amplitude ratios are also made for the particular cases. The physical constants for medium $M_{1}$ are taken as

$$
\begin{aligned}
& \lambda=2.238 \mathrm{~N} / \mathrm{m}^{2}, \quad \mu=3.68 \mathrm{~N} / \mathrm{m}^{2}, \quad \rho=8.965 \mathrm{~kg} / \mathrm{m}^{3}, \\
& c_{e}=1.04 \times 10^{3} \mathrm{Jkg}^{-1} \mathrm{deg}^{-1}, \quad K^{*}=2.4 \times 10^{2} \mathrm{Wm}^{-1} \mathrm{deg}^{-1}, \\
& T_{0}=293^{\circ} \mathrm{K}, \quad l=17.6 \mathrm{~N}, \quad \bar{\beta}=2.03 \mathrm{Nm}^{-1} \mathrm{deg}^{-1}, \quad \tau_{0}=.0068, \quad \tau_{l}=0.000813 .
\end{aligned}
$$

The physical constants for medium $M_{2}$ are as

$$
\begin{aligned}
& \bar{\lambda}=7.76 \mathrm{~N} / \mathrm{m}^{2}, \quad \bar{\mu}=3.28 \mathrm{~N} / \mathrm{m}^{2}, \quad \bar{\rho}=1.74 \mathrm{~kg} / \mathrm{m}^{3}, \\
& \bar{c}_{e}=1.021 \times 10^{3} \mathrm{Jkg}^{-1} \mathrm{deg}^{-1}, \quad \bar{K}^{*}=1.7 \times 10^{2} \mathrm{Wm}^{-1} \mathrm{deg}^{-1}, \\
& \bar{T}_{0}=296^{\circ} \mathrm{K}, \quad \bar{l}=2.05 \mathrm{~N}, \quad \bar{\beta}=2.68 \mathrm{Nm}^{-1} \mathrm{deg}^{-1}, \\
& \tau_{0}=0.00803, \quad \tau_{1}=0.0043 .
\end{aligned}
$$


We have used MATLAB 7.12.0.635(R2011a) to find amplitude ratios for the angle of incidence of the incident longitudinal wave (P), thermal wave (T) and incident transverse (SV-I and SV-II) waves. The amplitude ratios for reflected longitudinal waves reflected thermal waves reflected transverse waves in medium $M_{1}$ and corresponding transmitted waves in medium $M_{2}$ are shown graphically in Figs 2-33.

In all the figures the word CLS, CGL denote the values of amplitude ratios for couple stress generalized thermoelastic solids while the words WCLS, WCGL are used to correspond to thermoelastic solids in the context of Lord-Shulman and Green-Lindsay theories, respectively. The variations of the amplitude ratios $\left|Z_{i}\right|,(i=1,2, \ldots \ldots .8)$ of various reflected and transmitted waves for CLS, CGL, WCLS and WCGL with the angle of emergence $\theta_{0}$ of different cases of the incident wave (P-, T-, SV1- or SV2-) have been shown in Figs 2-33.

\subsection{Incident P- wave}

The variations of the absolute values of the amplitude ratios of various reflected and transmitted waves with the angle of emergence varying from $0^{\circ} \leq \theta_{0} \leq 90^{\circ}$ of incident P-wave have been shown in Figs $2-9$.

Figure 2 shows the variation of amplitude ratios of reflected P-wave with the angle of emergence for CLS, CGL, WCLS and WCGL. It is seen that the value of amplitude ratios increase with increase in the value of the angle of incidence for CLS and WCLS whereas the value of $\left|Z_{1}\right|$ decreases in $0^{\circ} \leq \theta_{0} \leq 14.8^{\circ}$ increases in $14.8^{\circ} \leq \theta_{0} \leq 26.6^{\circ}$ thereafter decreases smoothly with an increase in the v angle of the angle of emergence for CGL. The value of the amplitude ratio $\left|Z_{1}\right|$ is almost constant for WCGL. Figure 3 presents the variation of amplitude ratios of the reflected T-wave with the angle of incidence $\theta_{0}$ for various theories. It is noticed that the values of the amplitude ratio $\left|Z_{2}\right|$ decrease with an increase in the angle of incidence for CLS, CGL, WCLS and WCGL. The values of amplitude ratio $\left|Z_{2}\right|$ decrease sharply for WCLS for small values of the angle of incidence in comparison with CLS, CGL and WCGL.

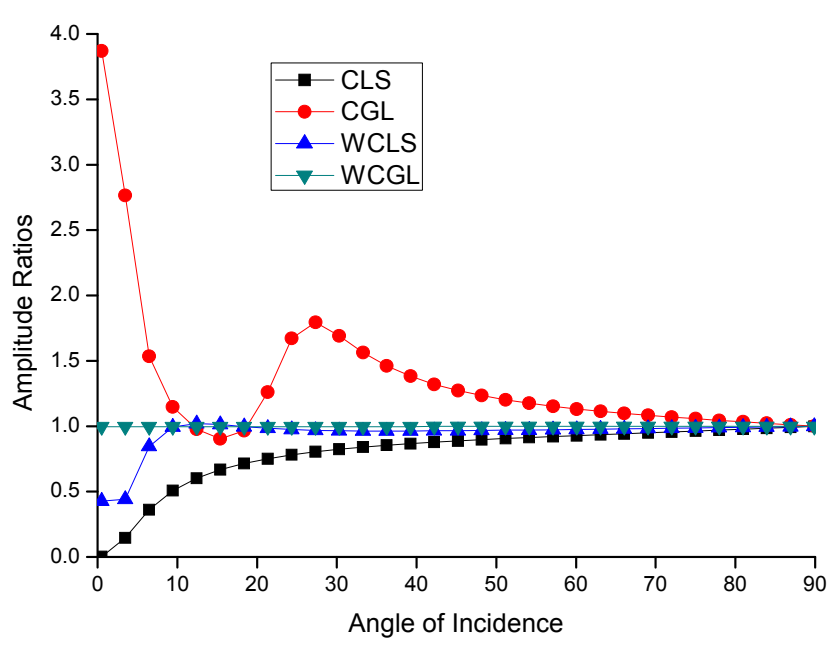

Fig.2. Variation of amplitude ratios of reflected $\mathrm{P}$-waves versus the angle of incidence.

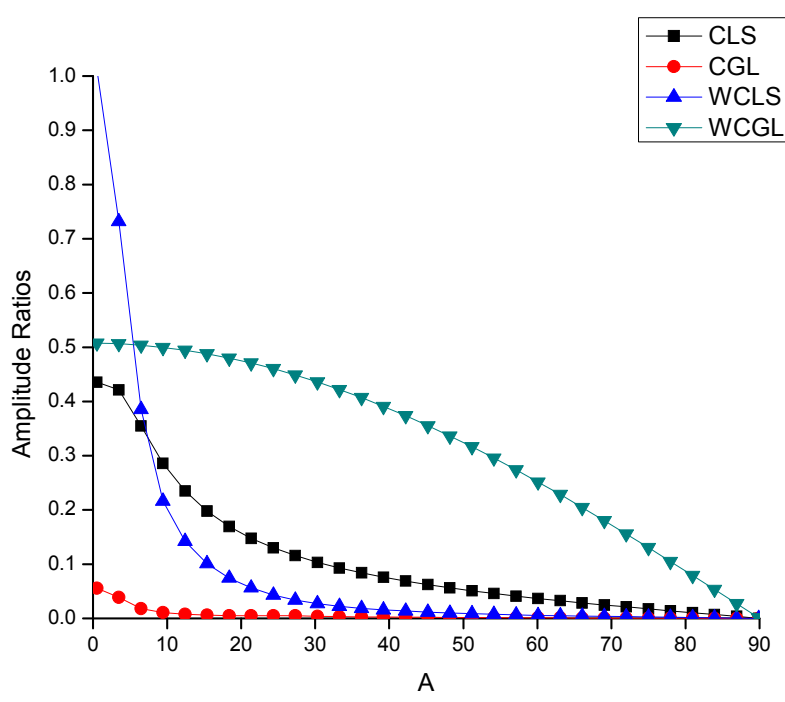

Fig.3. Variation of amplitude ratios of reflected $\mathrm{T}$-waves versus the angle of incidence. 
The variations of amplitude ratios of reflected SV1- wave with the angle of emergence for various theories are shown in Fig.4. The values of the amplitude ratio $\left|Z_{3}\right|$ decrease sharply for small values of the angle emergence thereafter decreases smoothly with an increase in the angle of emergence for all the theories. Figure 5 depicts that variation of amplitude ratios of transmitted SV2- wave with the angle of emergence. It is evident that the values of the amplitude ratio $\left|Z_{4}\right|$ decrease in $0^{\circ} \leq \theta_{0} \leq 19.6^{\circ}$ increase in $19.6^{\circ} \leq \theta_{0} \leq 19.8^{\circ}$ and decrease with an increase in the angle of emergence for CGL whereas it decreases for all values for the angle of emergence for CLS. The values of the amplitude ratios $\left|Z_{4}\right|$ are very low for WCLS and WCG in comparison with CLS and CGL.

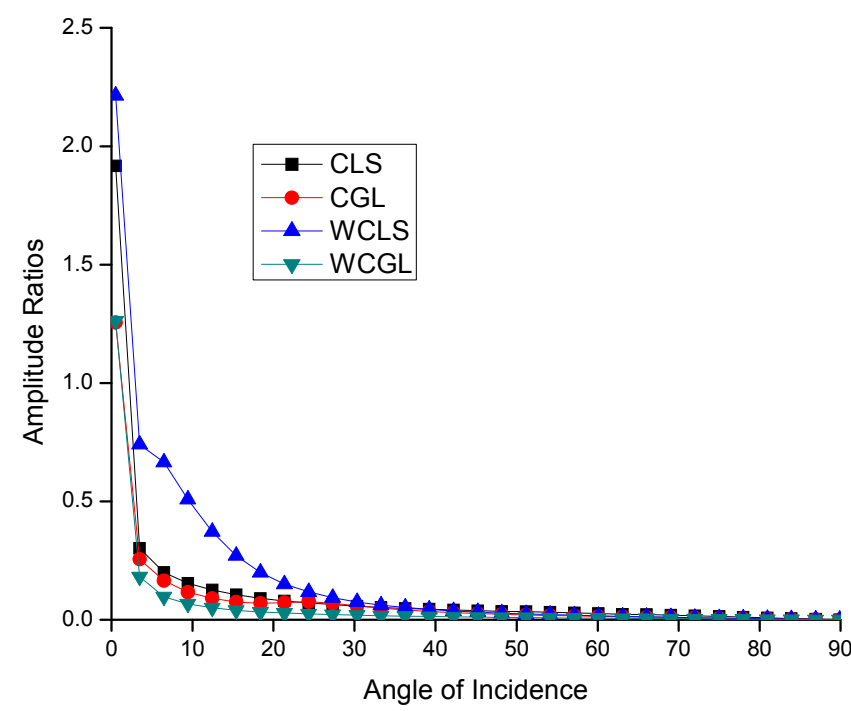

Fig.4. Variation of amplitude ratios of reflected SV1-waves versus the angle of incidence.

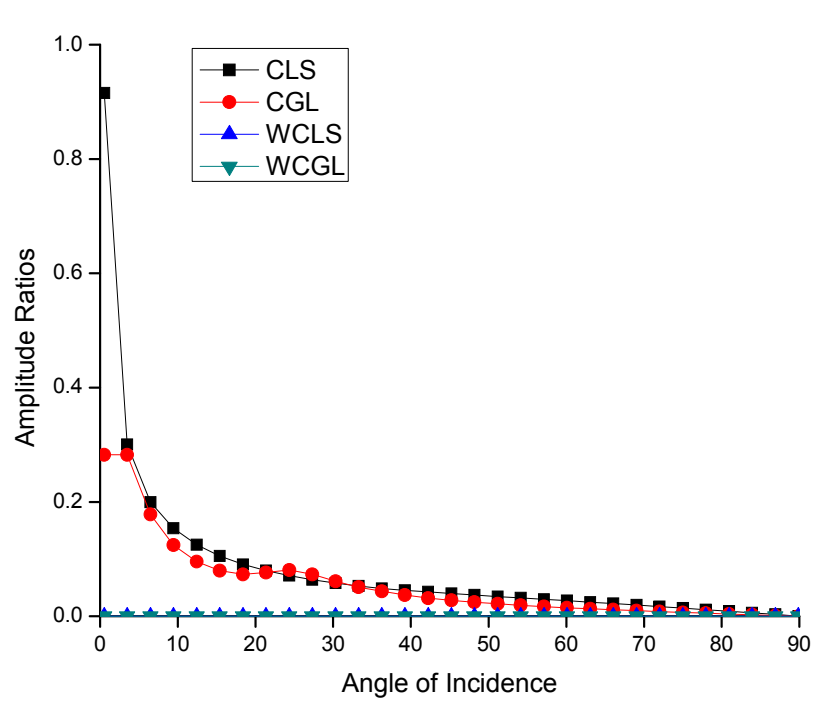

Fig.5. Variation of amplitude ratios of reflected SV2 -waves versus the angle of incidence.

The variations of amplitude ratios $\left|Z_{5}\right|$ (transmitted P-wave) for different theories CLS, CGL, WCLS and WCGL are shown in Fig.6. It can be seen that the values of $\left|Z_{5}\right|$ oscillate for small values of the angle of incidence then decrease smoothly for CGL whereas $\left|Z_{5}\right|$ decrease with the increase in the angle of incidence for CLS, WCGL and WCLS. Figure 7 shows the variations of amplitude ratios $\left|Z_{6}\right|$ of transmitted T-wave for CLS, CGL, WCLS and WCGL with respect to the angle of emergence. The values of $\left|Z_{6}\right|$ oscillate for small values of the angle of incidence, decrease for large values of the angle of incidence for CGL whereas the values of $\left|Z_{6}\right|$ increase for small values of the angle of incidence, decrease thereafter with an increase in the values of the angle of incidence for WCLS whereas the values of amplitude ratios $\left|Z_{6}\right|$ decrease with an increase in the angle of incidence for CLS and WCGL. 


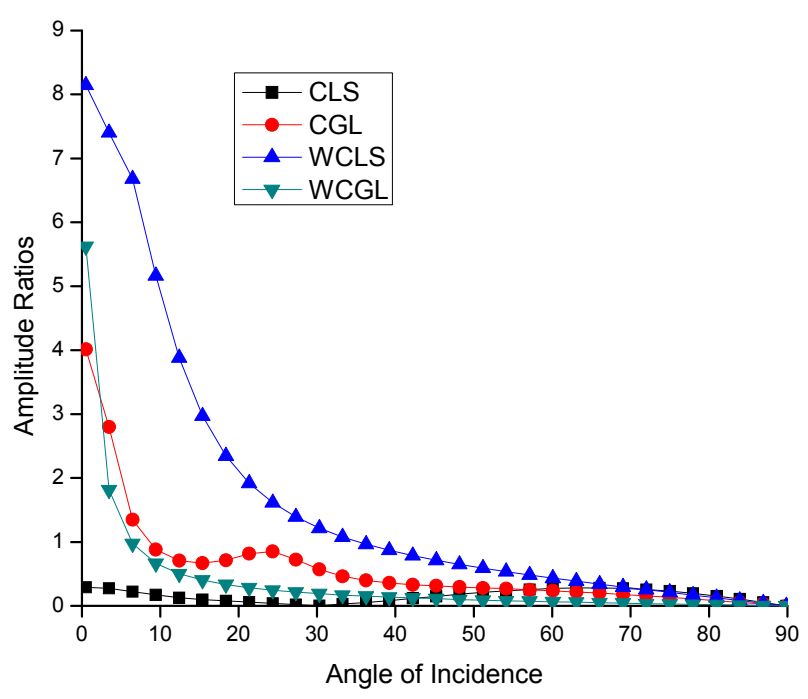

Fig.6. Variation of amplitude ratios of transmitted $\mathrm{P}$-waves versus the angle of incidence.

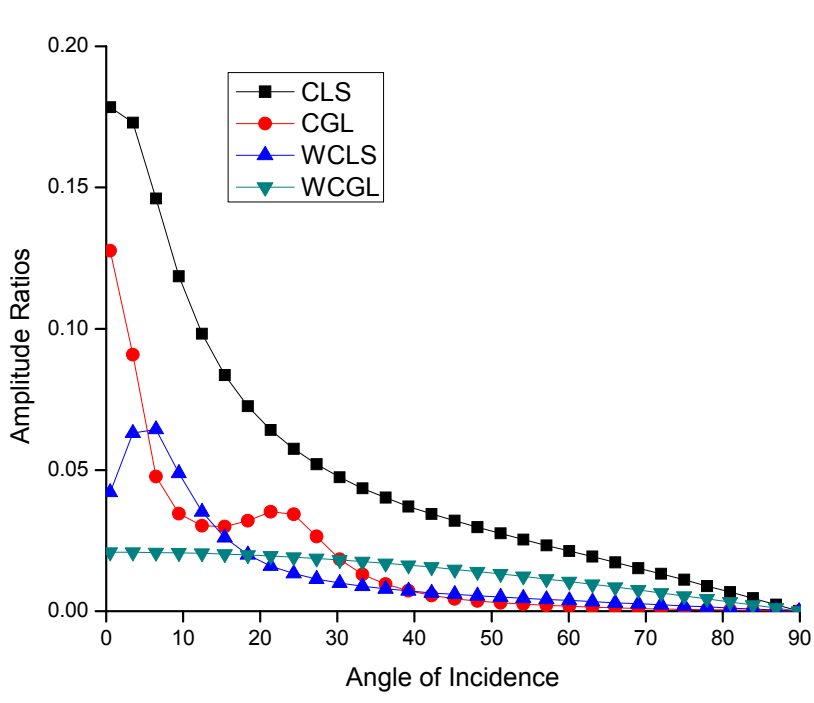

Fig.7. Variation of amplitude ratios of transmitted $\mathrm{T}$-waves versus the angle of incidence.

Figure 8 represents the variations of amplitude ratios $\left|Z_{7}\right|$ of transmitted SV1- wave with respect to the angle of emergence. It is noticed that the values of $\left|Z_{7}\right|$ increase in $0^{\circ} \leq \theta_{0} \leq 10^{\circ}$ for WCLS and in $0^{\circ} \leq \theta_{0} \leq 30^{\circ}$ WCGL and the values of the amplitude ratio $\left|Z_{7}\right|$ for WCLS are always greater than WCGL, CLS and CGL. Figure 9 depicts the variations of the amplitude $\left|Z_{8}\right|$ of transmitted SV2-wave. It is noticed that the values of $\left|Z_{8}\right|$ decrease sharply in $0^{\circ} \leq \theta_{0} \leq 18^{\circ}$ increase in $18^{\circ} \leq \theta_{0} \leq 52^{\circ}$ and decrease thereafter for the remaining values of the angle of incidence for CLS whereas $0^{o} \leq \theta_{0} \leq 11^{o}$ increases in $11^{\circ} \leq \theta_{0} \leq 26^{\circ}$ thereafter decrease smoothly with an increase in the values of the angle of incidence for CGL.

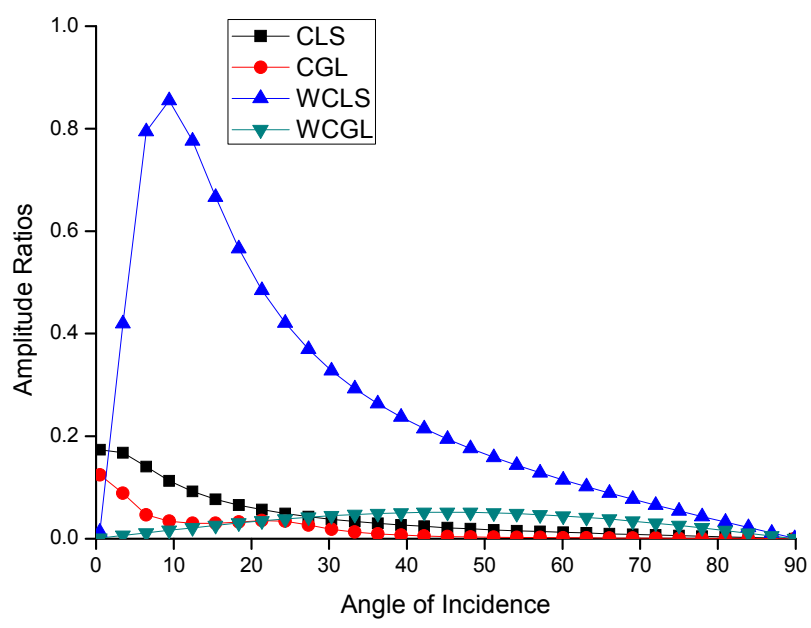

Fig.8. Variation of amplitude ratios of transmitted SV1-waves versus the angle of incidence.

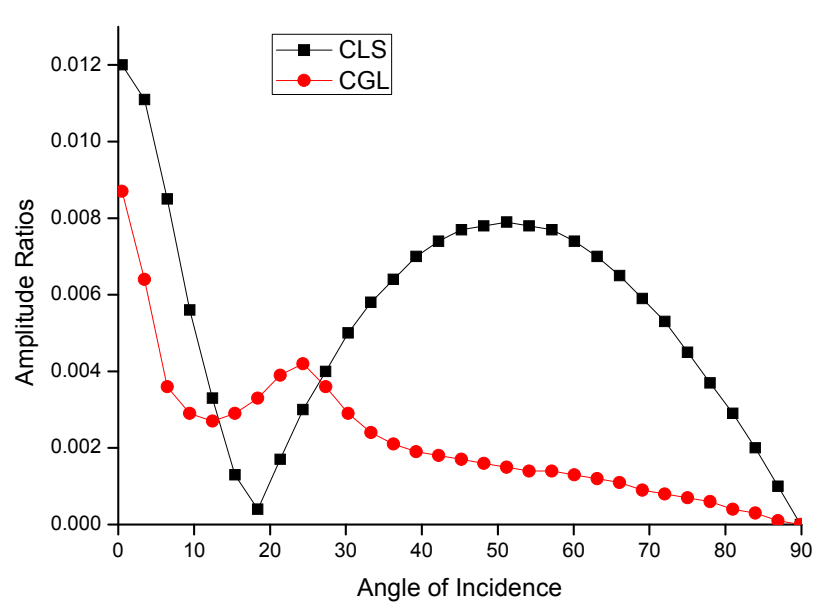

Fig.9. Variation of amplitude ratios of transmitted SV2 -waves versus the angle of incidence. 


\subsection{Incident T-wave}

It is clear from Fig. 10 that the values of the amplitude ratio $\left|Z_{l}\right|$ increase with an increase in the angle of incidence $\left(\theta_{0}\right)$ and decrease for large values of the angle of incidence for WCLS. The values of the amplitude ratio $\left|Z_{1}\right|$ increase in $0^{\circ} \leq \theta_{0} \leq 43^{\circ}$ then decrease with an increase in the angle for CGL. The values of the amplitude ratio $\left|Z_{l}\right|$ decrease with an increase in the angle of emergence for CLS and WCGL. Figure 11 indicates that the values of the amplitude ratio $\left|Z_{2}\right|$ increase with an increase in the angle of emergence and then decrease for large values of the angle of emergence for CGL whereas a reverse behavior is observed for CLS. The values of amplitude ratios $\left|Z_{2}\right|$ increase for all values of the angle of incidence for WCGL. Figure 12 depicts the variation of the amplitude ratio for reflected SV1- wave for CLS, CGL, WCLS and WCGL. It shows that the values of the amplitude ratio $\left|Z_{3}\right|$ decrease sharply in the range $0^{\circ} \leq \theta_{0} \leq 9^{\circ}$ for CLS, WCLS and WCGL whereas the values of the amplitude ratio $\left|Z_{3}\right|$ decrease smoothly for the remaining values of the CGL angle of emergence for WCGL and CLS and an oscillatory behavior is observed for CGL.

Figure 13 shows the variations of the amplitude ratio $\left|Z_{4}\right|$ is similar to $\left|Z_{3}\right|$ with a difference in magnitude values. Figure 14 shows the variations of the amplitude ratio $\left|Z_{5}\right|$ for transmitted P-wave. The values of the amplitude ratio $\left|Z_{5}\right|$ decrease sharply in the range $0^{\circ} \leq \theta_{0} \leq 3.5^{\circ}$, increase in the range $3.5^{\circ} \leq \theta_{0} \leq 46^{\circ}$, and decrease thereafter as the angle of incidence increases for CGL whereas the values of the amplitude ratio $\left|Z_{5}\right|$ decrease smoothly with an increase in the values of the angle of incidence for CLS. The values of the amplitude ratio $\left|Z_{5}\right|$ are very low for WCGL with an increase in the angle of emergence in comparison with other theories. Figure 15 shows the variations of the amplitude ratio of $\left|Z_{6}\right|$ for transmitted T-wave. The values of amplitude ratios $\left|Z_{6}\right|$ increase in $0^{\circ} \leq \theta_{0} \leq 43^{\circ}$ and decrease for the remaining values of the angle of incidence for CGL whereas the values of the amplitude ratio $\left|Z_{6}\right|$ decrease smoothly for CLS and WCGL. Figure 16 represents the variations of the amplitude ratio $\left|Z_{7}\right|$ for SV1- transmitted wave. It can be seen that the behavior of values of the amplitude ratio $\left|Z_{7}\right|$ is different for WCGL, WCLS and CLS, CGL with an increase in the values of the angle of incidence. Figure 17 depicts the variations of the amplitude ratio $\left|Z_{8}\right|$ for SV2- transmitted wave. It can be seen that the values of $\left|Z_{8}\right|$ are always higher for CGL than CLS.

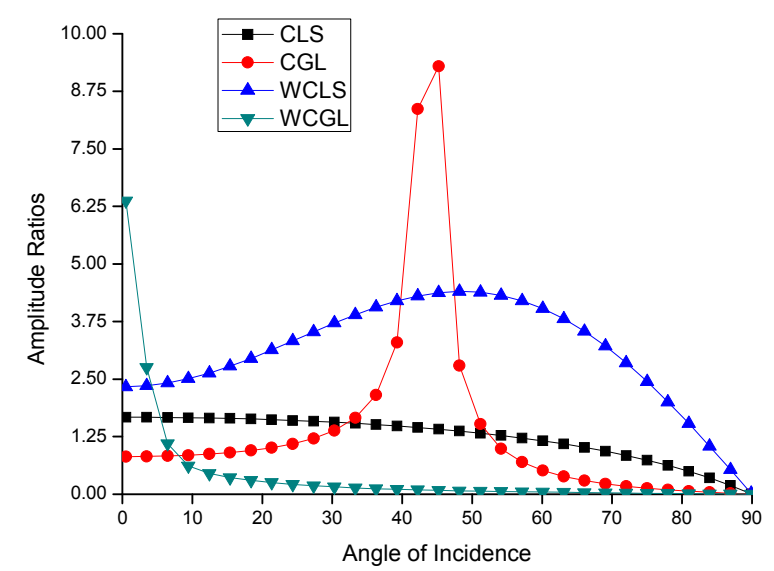

Fig.10. Variation of amplitude ratios of reflected $\mathrm{P}$-waves versus the angle of incidence.

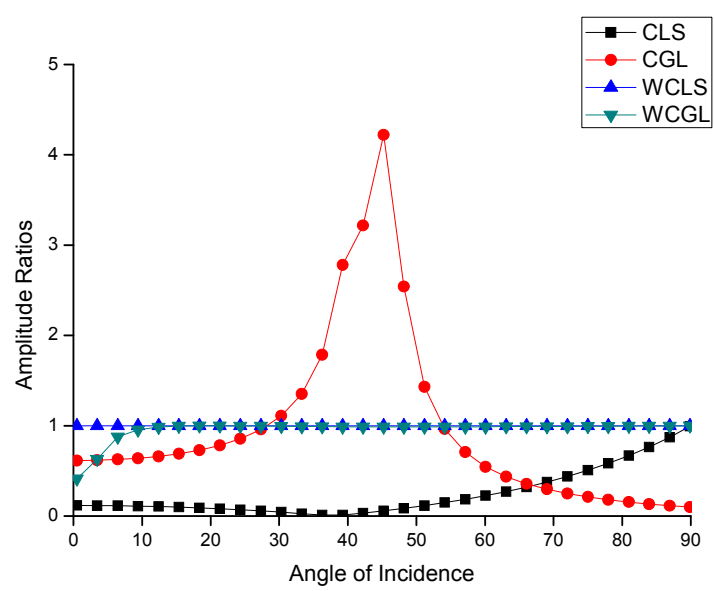

Fig.11. Variation of amplitude ratios of reflected $\mathrm{T}$-waves versus the angle of incidence. 


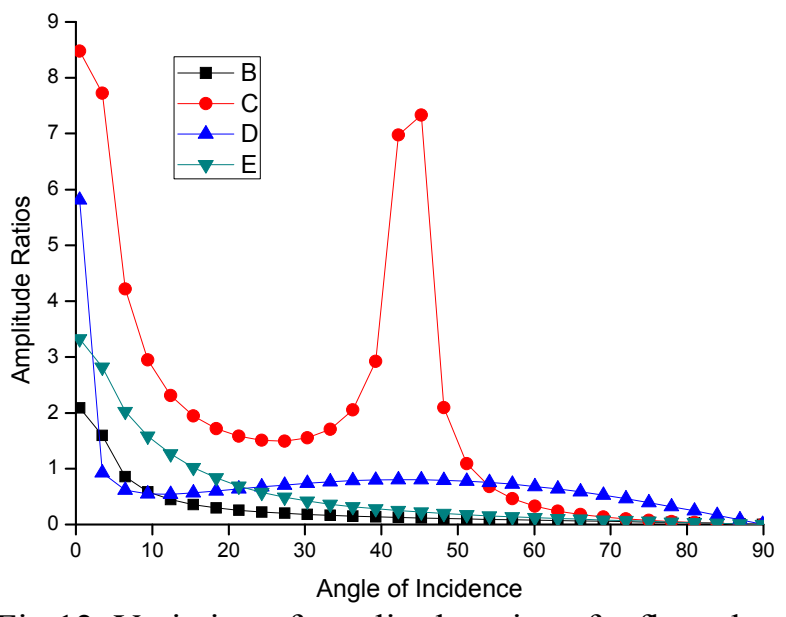

Fig.12. Variation of amplitude ratios of reflected SV1-waves versus the angle of incidence.

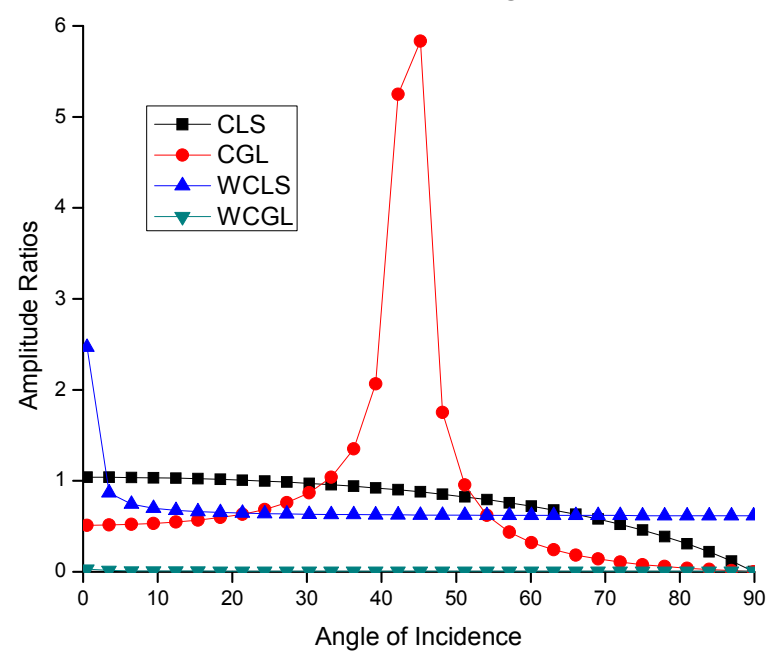

Fig.14. Variation of amplitude ratios of transmitted P-waves versus the angle of incidence.

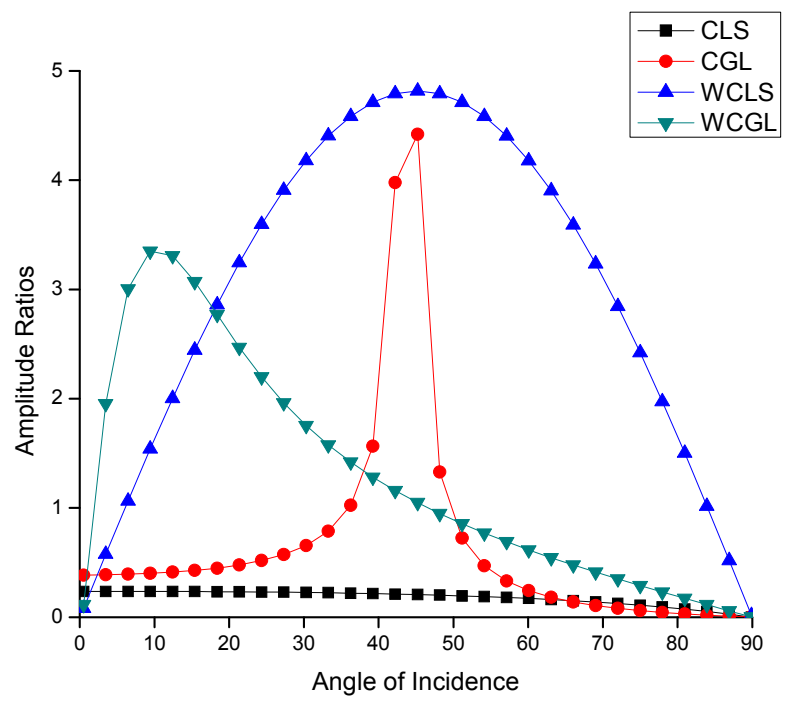

Fig.16. Variation of amplitude ratios of transmitted SV1-waves versus the angle of incidence.

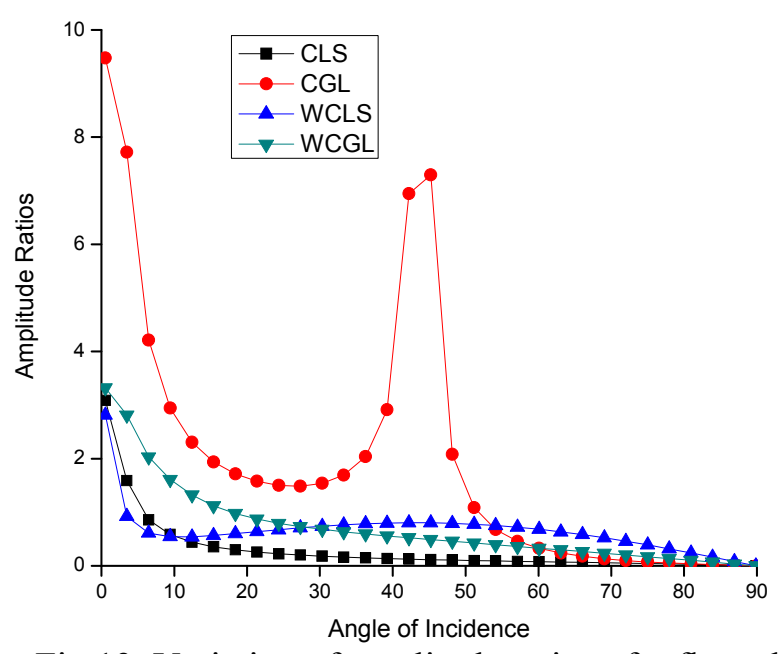

Fig.13. Variation of amplitude ratios of reflected SV2-waves versus the angle of incidence.

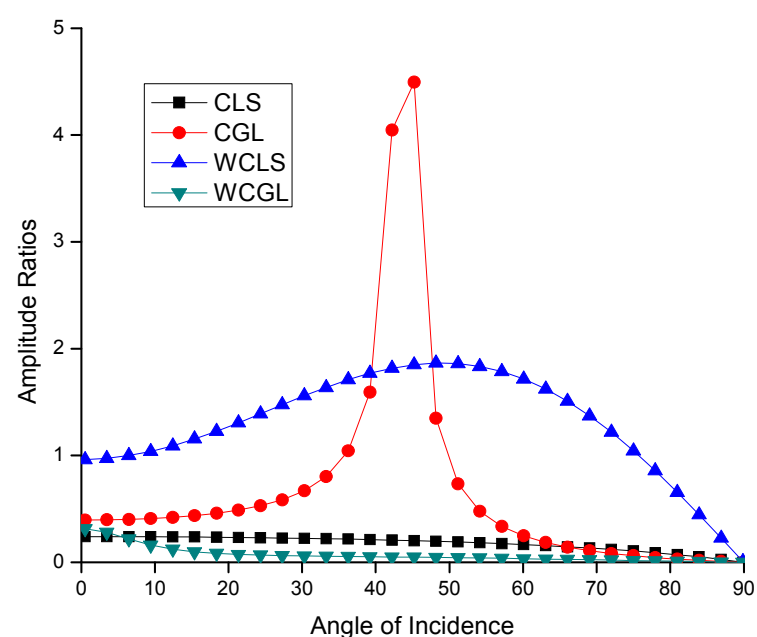

Fig. 15. Variation of amplitude ratios of transmitted $\mathrm{T}$-waves versus the angle of incidence.

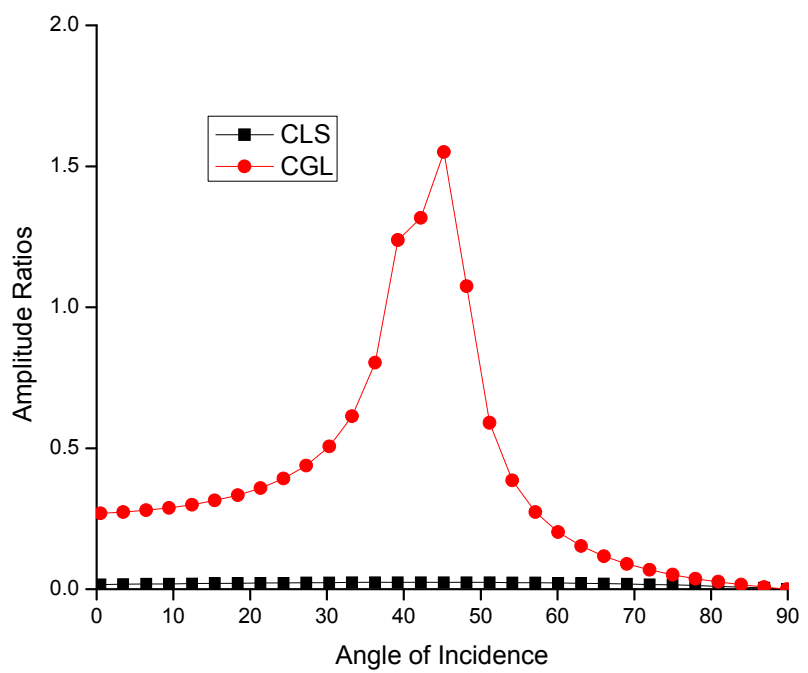

Fig.17. Variation of amplitude ratios of transmitted SV2-waves versus the angle of incidence. 


\subsection{Incident SV-1 Wave}

Figure 18 shows the variations of the amplitude ratio $\left|Z_{l}\right|$ for reflected $\mathrm{P}$-wave. The values of the amplitude ratio $\left|Z_{1}\right|$ decrease in the range $0^{\circ} \leq \theta_{0} \leq 10^{\circ}$ sharply, then decrease smoothly with an increase in the values of the angle of incidence for CLS, CGL and WCGL whereas with the values of amplitude ratios decrease smoothly with an increase in the values of the angle of incidence for WCLS. Figure 19 shows the variations of the amplitude ratio $\left|Z_{2}\right|$ for reflected P-wave. It is seen that the values of amplitude ratios decrease sharply for small values of the angle of incidence for CLS and CGL in comparison with WCLS and WCGL. Figure 20 represents the variations of the amplitude ratio $\left|Z_{3}\right|$ of reflected SV1- wave. It can be observed that the behavior of $\left|Z_{3}\right|$ is similar for CGL, CLS and for WCLS and WCGL. But the behavior of $\left|Z_{3}\right|$ is reverse for CLS, CGL and WCLS, WCGL. Figure 21 depicts the variations of the amplitude ratio $\left|Z_{4}\right|$ of reflected SV2- wave. The values of $\left|Z_{4}\right|$ decrease with an increase in the values of the angle of incidence for CLS, CGL, WCLS whereas the values of $\left|Z_{4}\right|$ increase for small values of the angle of incidence and decrease with an increase in the values of the angle of incidence for WCGL. Figure 22 shows the variations of the amplitude ratio $\left|Z_{5}\right|$ for transmitted P-wave. The behavior of the values of the amplitude ratio $\left|Z_{5}\right|$ is similar for WCLS and WCGL whereas a reverse behavior is observed for CLS, CGL. Figure 23 represents the variations of the amplitude ratio $\left|Z_{6}\right|$ of transmitted T-wave. The values of the amplitude ratio $\left|Z_{6}\right|$ decrease in the range $0^{\circ} \leq \theta_{0} \leq 4^{\circ}$, increase in $4^{o} \leq \theta_{0} \leq 10^{\circ}$ and decrease again with an increase in the values of the angle of incidence for WCGL. The values of the amplitude ratio $\left|Z_{6}\right|$ decrease in the range $0^{\circ} \leq \theta_{0} \leq 6.4^{\circ}$, increase in $6.4^{\circ} \leq \theta_{0} \leq 8.4^{\circ}$ again decrease smoothly with an increase in the angle of incidence for WCLS whereas the values of $\left|Z_{6}\right|$ decrease with an increase in the angle of emergence for CLS and CGL. Figures 2425 represents the variations of the amplitude ratio $\left|Z_{7}\right|$ and $\left|Z_{8}\right|$ of transmitted SV1 and SV2- waves, respectively. It is observed that the behavior of the values of the amplitude ratio $\left|Z_{7}\right|$ and $\left|Z_{8}\right|$ are similar with a difference in the magnitude values.

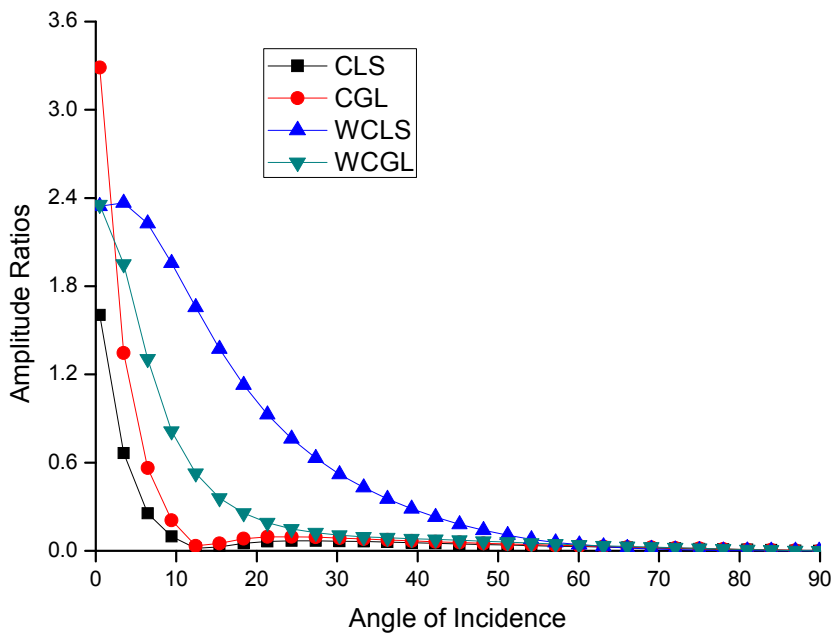

Fig.18. Variation of amplitude ratios of reflected $\mathrm{P}$-waves versus the angle of incidence.

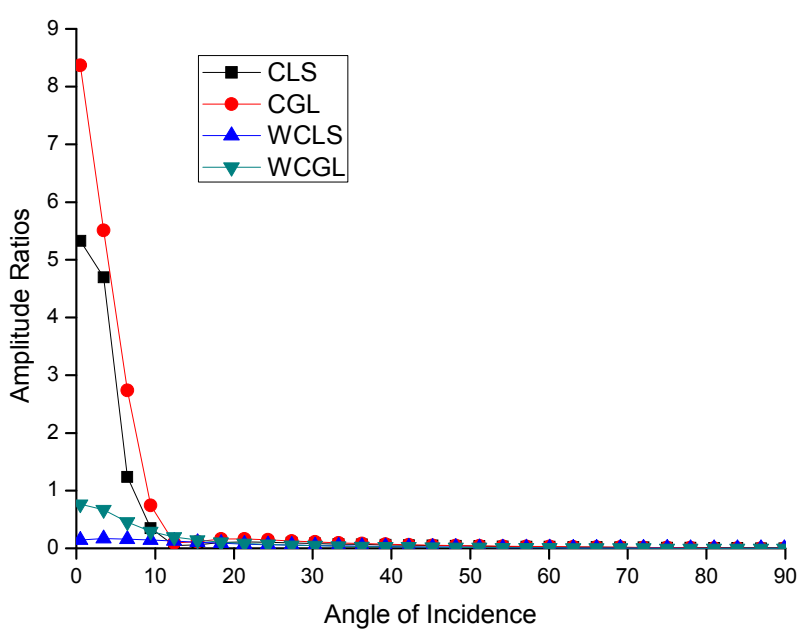

Fig.19. Variation of amplitude ratios of reflected $\mathrm{T}$-waves versus the angle of incidence. 


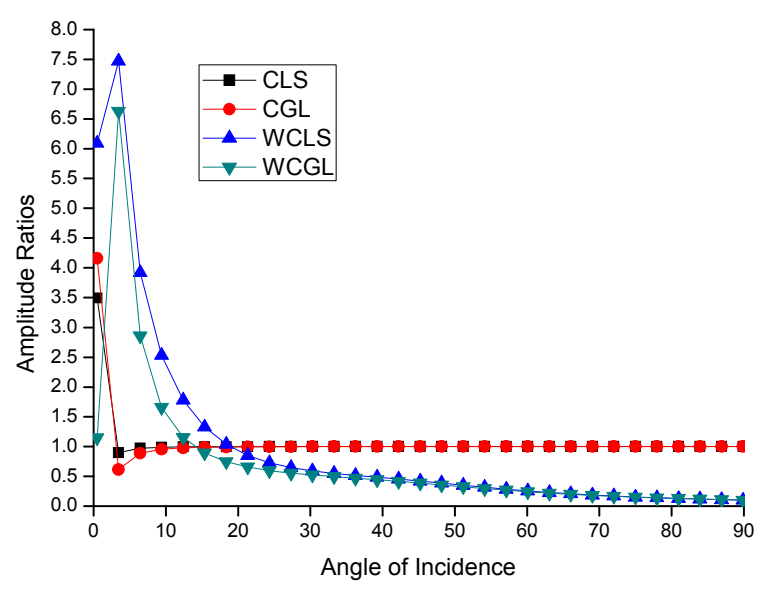

Fig.20. Variation of amplitude ratios of reflected SV1-waves versus the angle of incidence.

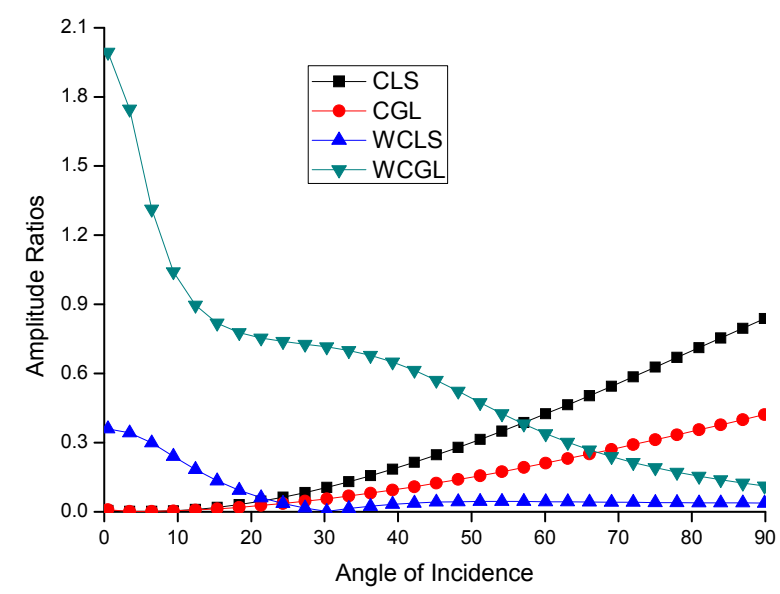

Fig.22. Variation of amplitude ratios of transmitted $\mathrm{P}$-waves versus the angle of incidence.

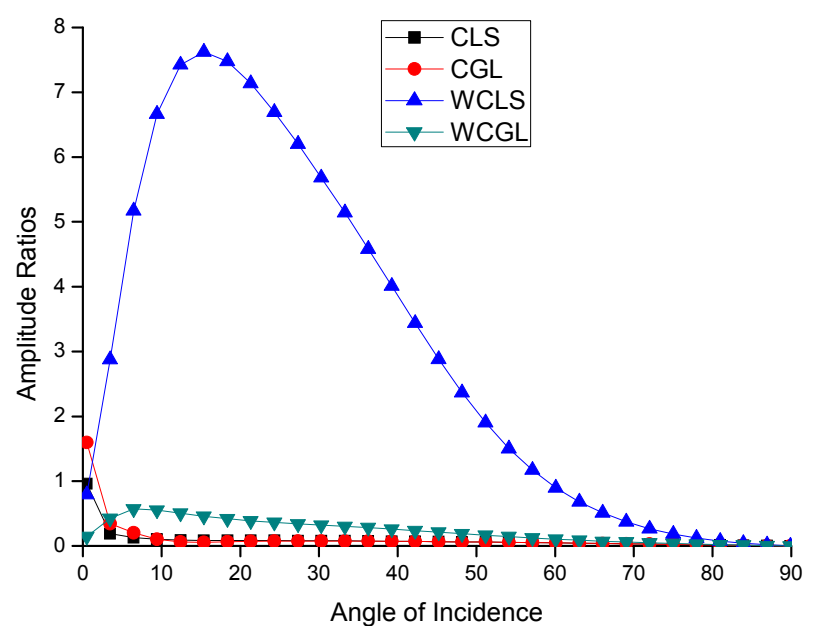

Fig.24. Variation of amplitude ratios of transmitted SV1-waves versus the angle of incidence.

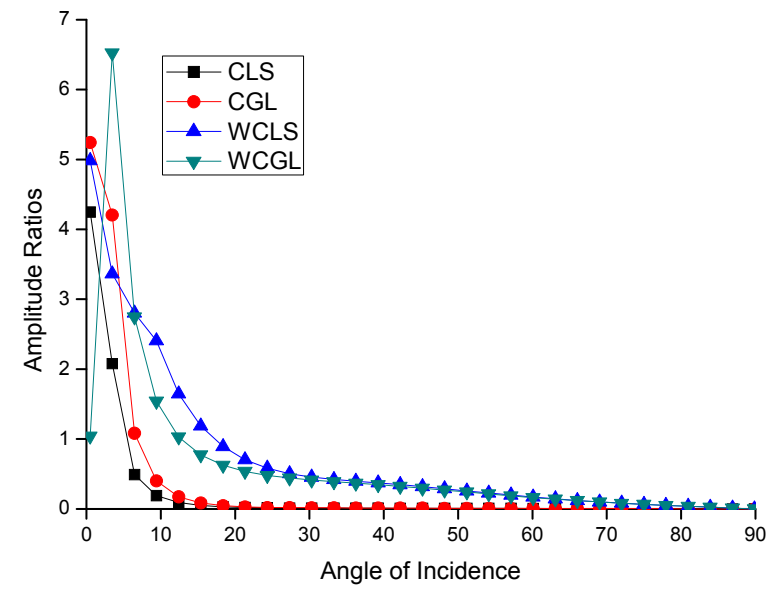

Fig.21. Variation of amplitude ratios of reflected SV2-waves versus the angle of incidence.

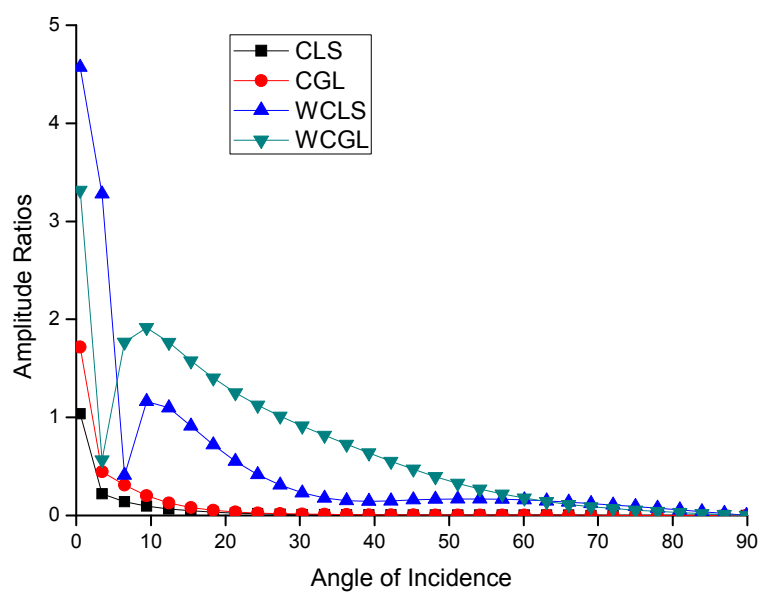

Fig.23. Variation of amplitude ratios of transmitted $\mathrm{T}$-waves versus the angle of incidence.

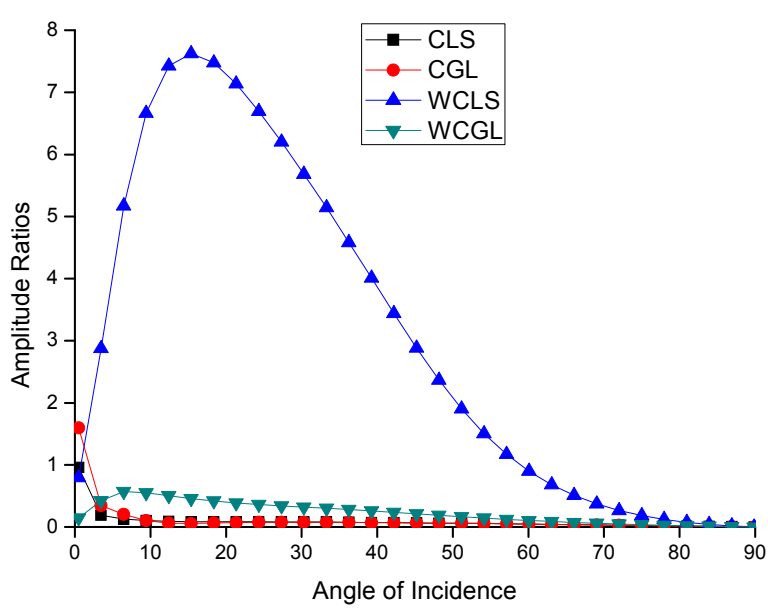

Fig.25. Variation of amplitude ratios of transmitted SV2-waves versus the angle of incidence. 


\subsection{Incident SV2- wave}

Figures 26-33 show the variations of the amplitude ratio $\left|Z_{i}\right|(i=1,2 \ldots \ldots \ldots . .8$.) for reflected and transmitted P-, T-, SV1- and SV2- waves. Figures 26 depicts the variations of the amplitude ratio $\left|Z_{1}\right|$ of reflected P-wave. The values of the amplitude ratio $\left|Z_{1}\right|$ increase in $0^{\circ} \leq \theta_{0} \leq 3^{\circ}$ for WCGL whereas the reverse behavior is observed for CLS, WCLS and CGL. The values of the amplitude ratio $\left|Z_{l}\right|$ decrease smoothly for the remaining values of the angle of incidence and the values of the amplitude ratios remain low for WCLS in comparison to CLS, CGL and WCGL. Figures 27- 28 show the variation of amplitude ratio of reflected T-wave and SV1- wave. It is observed the behavior of values of the amplitude ratio is similar with a difference in the magnitude values for different values of the angle of incidence. From Fig.29 it is observed that the values of the amplitude ratio $\left|Z_{4}\right|$ decrease sharply for small values of the angle incidence for WCLS and WCGL whereas the values of $\left|Z_{4}\right|$ are almost constant for CLS and CGL for different values of the angle of incidence. Figure 30 shows the variation of the values of the amplitude ratio $\left|Z_{5}\right|$ of transmitted P-wave. It is observed that the values of the amplitude ratio $\left|Z_{5}\right|$ decrease sharply for small values of the angle of incidence and decrease smoothly with an increase in the values of the angle of incidence for CLS and CGL. Figure 31 shows the variation of the values of the amplitude ratio $\left|Z_{6}\right|$ of transmitted T-wave. The values of the amplitude ratio $\left|Z_{6}\right|$ decrease with an increase in the values of the angle of incidence for CLS, CGL and WCGL whereas the values of the amplitude ratio $\left|Z_{6}\right|$ decrease in $0^{\circ} \leq \theta_{0} \leq 3^{\circ}$, increase in $3^{o} \leq \theta_{0} \leq 16^{\circ}$ and decrease again with an increase in the values of the angle of incidence for WCLS. Figure 32 depicts the variation of the values of the amplitude ratio $\left|Z_{7}\right|$ of transmitted SV1-wave. The values of the amplitude ratio decrease with an increase in the values of the angle of incidence for CLS, CGL, WCLS and WCGL. Figure 33 presents the variation of the values of the amplitude ratio $\left|Z_{8}\right|$ of transmitted SV2-wave. It is observed that the behavior of the amplitude ratio $\left|Z_{8}\right|$ is similar CLS and CGL but the values of $\left|Z_{8}\right|$ remain higher for CGL than CLS.

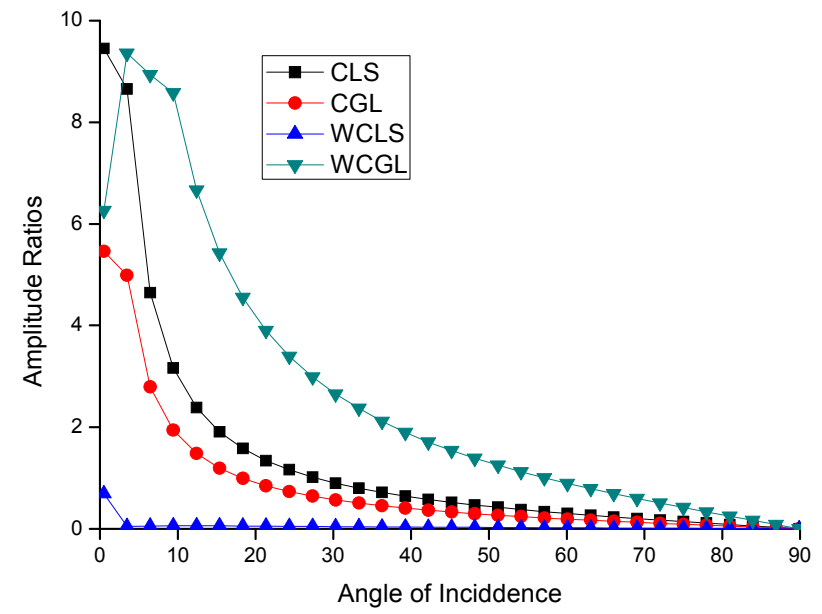

Fig.26. Variation of amplitude ratios of reflected P-waves versus the angle of incidence.

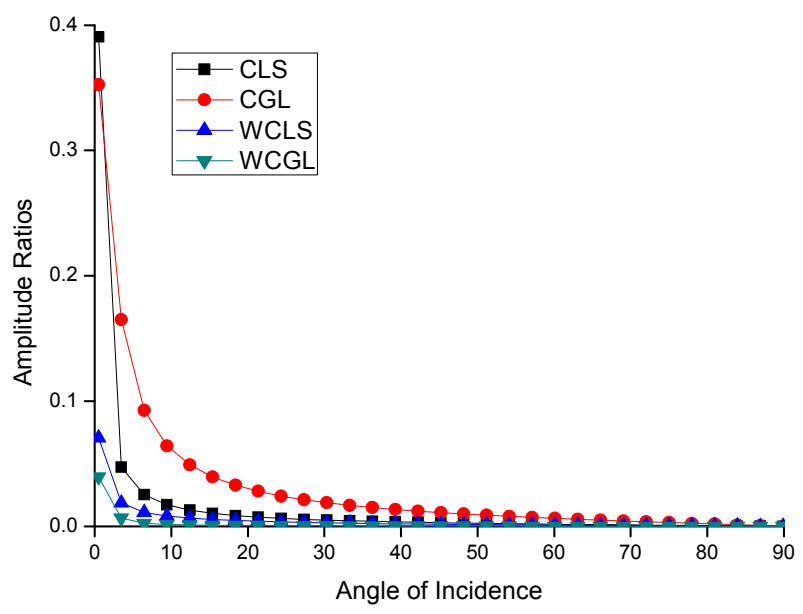

Fig.27. Variation of amplitude ratios of reflected $\mathrm{T}$-waves versus the angle of incidence. 


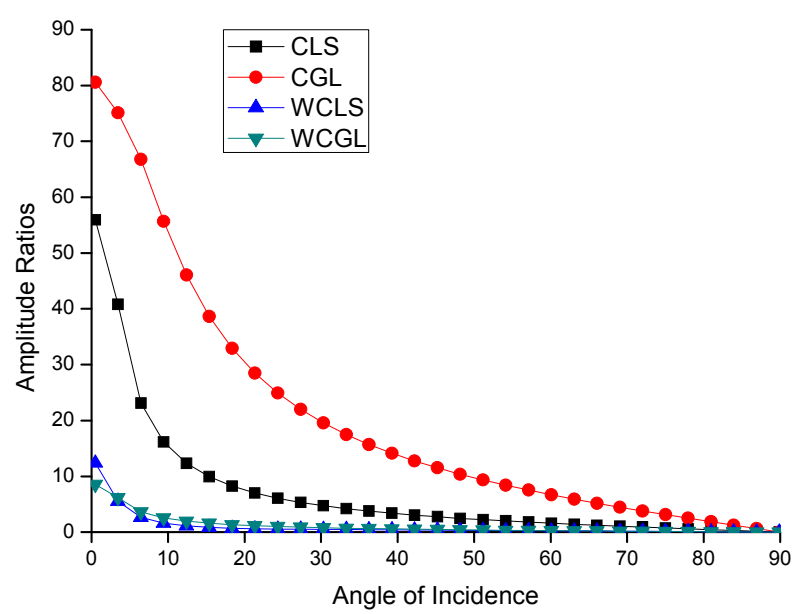

Fig.28. Variation of amplitude ratios of reflected SV1-waves versus the angle of incidence.

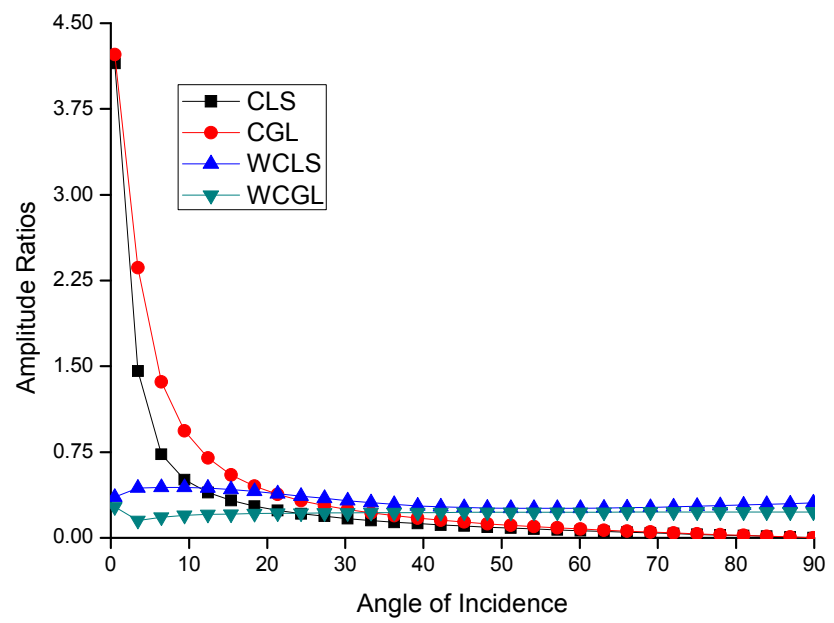

Fig.30. Variation of amplitude ratios of transmitted $\mathrm{P}$-waves versus the angle of incidence.

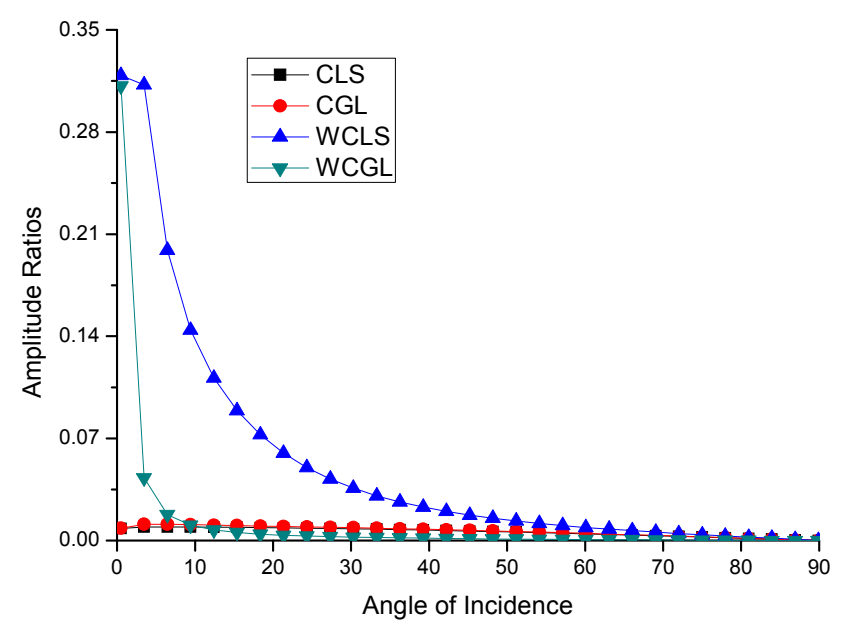

Fig.32. Variation of amplitude ratios of transmitted SV1-waves versus the angle of incidence.

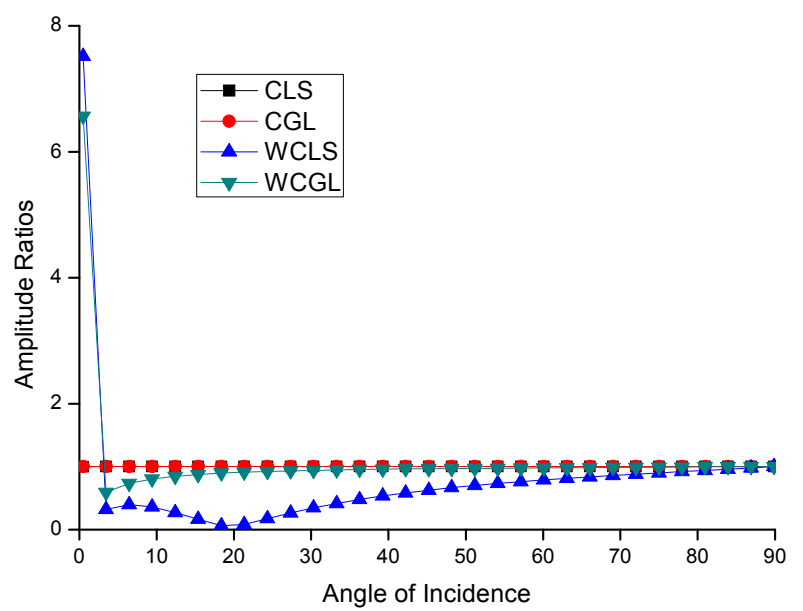

Fig.29. Variation of amplitude ratios of reflected SV2-waves versus the angle of incidence.

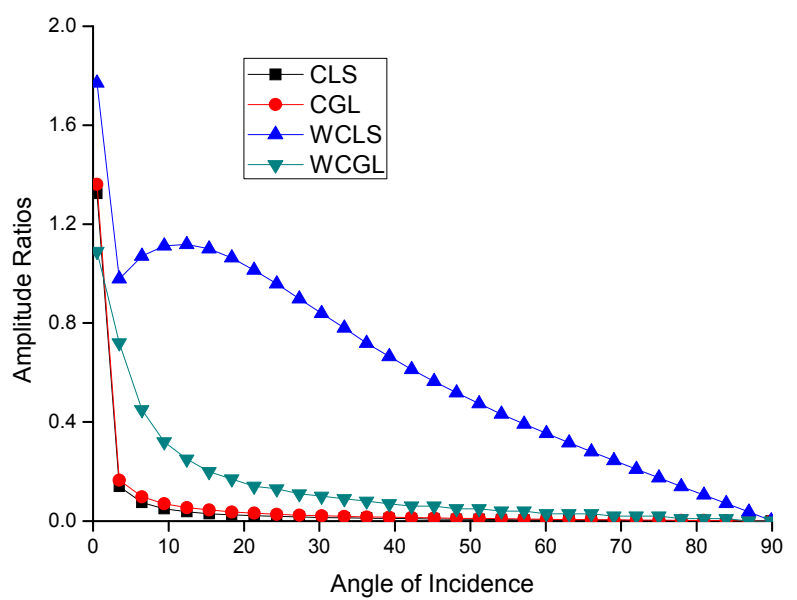

Fig.31. Variation of amplitude ratios of transmitted $\mathrm{T}$-waves versus the angle of incidence.

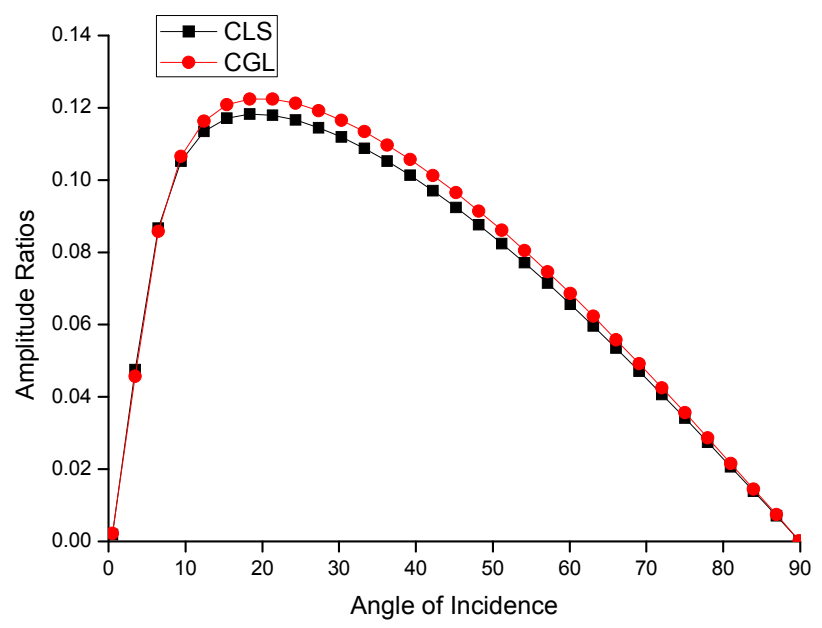

Fig.33. Variation of amplitude ratios of transmitted SV2- waves versus the angle of incidence. 


\section{Conclusion}

The expressions of amplitude ratios of reflection and transmission of plane waves have been obtained at the interface of two modified couple stress generalized thermoelastic solids. Some particular cases of interest are derived from the present investigation. It is observed that the couple stress effect plays an important role on the reflection and transmission phenomena. It is observed that the values of the amplitude ratio of reflected and transmitted P-, T- SV1- and SV2- are oscillatory when P- wave is incident. Mixed behavior is observed for the values of the amplitude ratio of reflected and transmitted P-, T- SV1- and SV2- waves when T- and SV1- waves are incident. The values of reflected and transmitted P-, T-, SV1- and SV2- oscillate when SV2- wave is incident. The model studied in the present paper may be helpful for experimental scientists/seismologists working in various fields such as earthquake estimation and exploration of mineral ores present in the earth's crust.

\section{Nomenclature}

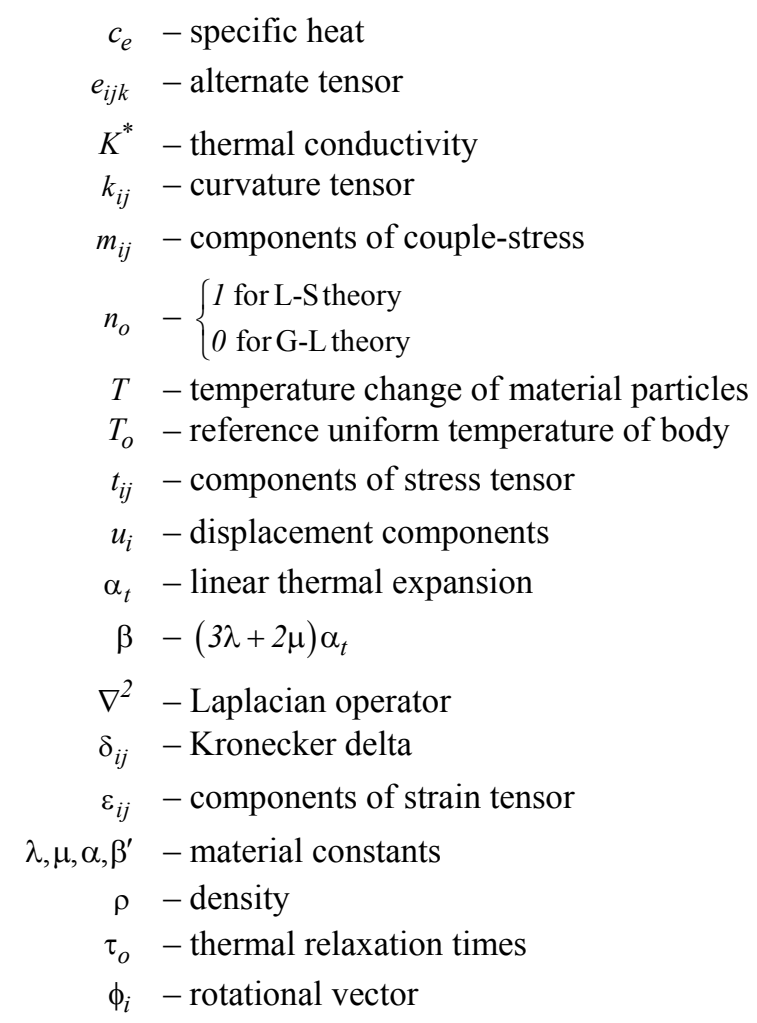

\section{References}

[1] Cosserat E. and Cosserat F. (1909): Theorie des Corps Deformables. - Paris: Hermann et Fils.

[2] Toupin R.A. (1962): Elastic materials with couple-stresses. - Arch. Ration Mech. Analysis, vol.11, No.1, pp.385414.

[3] Mindlin R.D. and Tiersten H.F. (1962): Effects of couple-stresses in linear elasticity. - Arch. Ration Mech. Analysis, vol.11, pp.415-448.

[4] Fleck N.A. and Hutchinson J.W. (1997): Strain gradient plasticity. - Advances in Applied Mechanics, vol.33, pp.295-361, Academic Press. 
[5] Yang F., Chong A.C.M., Lam D.C.C. and Tong P. (2002): Couple stress based strain gradient theory for elasticity. - International Journal of Solids and Structures, vol.39, No.10, pp.2731-2743.

[6] Ma H., Gao X. and Reddy J. (2008): A microstructure-dependent Timoshenko beam model based on a modified couple stress theory. - Journal of the Mechanics and Physics of Solids, vol.56, No.12, pp.3379-3391. doi:10.1016/j.jmps.2008.09.007

[7] Park S.K. and Gao X.-L. (2008): Micromechanical modeling of honeycomb structures based on a modified couple stress theory. - Mechanics of Advanced Materials and Structures, vol.15, No.8, pp.574-593.

[8] Yin L., Qian Q., Wang L. and Xia W. (2010): Vibration analysis of microscale plates based on modified couple stress theory. - Acta Mechanica Solida Sinica, vol.23, No.5, pp.386-393.

[9] Şimşek M. (2010): Dynamic analysis of an embedded microbeam carrying a moving microparticle based on the modified couple stress theory. - International Journal of Engineering Science, vol.48, No.12, pp.1721-1732.

[10] Jomehzadeh E., Noori H.R. and Saidi A.R. (2011): The size-dependent vibration analysis of micro-plates based on a modified couple stress theory. - Physica E: Low-Dimensional Systems and Nanostructures, vol.43, No.4, pp.877883.

[11] Ma H.M., Gao X.-L. and Reddy J. N. (2011): A non-classical Mindlin plate model based on a modified couple stress theory. - Acta Mechanica, vol.220, No.1-4, pp.217-235.

[12] Akgöz B. and Civalek Ö. (2012): Free vibration analysis for single-layered graphene sheets in an elastic matrix via modified couple stress theory. - Materials and Design, vol.42, pp.164-171.

[13] Nateghi A., Salamat-Talab M., Rezapour J. and Daneshian B. (2012): Size dependent buckling analysis of functionally graded micro beams based on modified couple stress theory. - Applied Mathematical Modelling, vol.36, No.10, pp.4971-4987.

[14] Chen W. and Li X. (2013): A new modified couple stress theory for anisotropic elasticity and microscale laminated Kirchhoff plate model. - Archive of Applied Mechanics, vol.84, No.3, 323-341. doi:10.1007/s00419-0130802-1.

[15] Gao X.-L., Huang J.X. and Reddy J.N. (2013): A non-classical third-order shear deformation plate model based on a modified couple stress theory. - Acta Mechanica, vol.224, No.11, pp.2699-2718.

[16] Ansari R., Faghih Shojaei M., Mohammadi V., Gholami R. and Darabi M.A. (2014): Nonlinear vibrations of functionally graded Mindlin microplates based on the modified couple stress theory. - Composite Structures, vol.114, pp.124-134.

[17] Kahrobaiyan M.H., Asghari M. and Ahmadian M.T. (2014): A Timoshenko beam element based on the modified couple stress theory. - International Journal of Mechanical Sciences, vol.79, pp.75-83. doi:10.1016/j.ijmecsci.2013.11.014

[18] Mohammad Abadi M. and Daneshmehr A.R. (2014): An investigation of modified couple stress theory in buckling analysis of micro composite laminated Euler-Bernoulli and Timoshenko beams. - International Journal of Engineering Science, vol.75, 40-53.

[19] Mohammad Abadi M. and Daneshmehr A.R. (2014): Size dependent buckling analysis of microbeams based on modified couple stress theory with high order theories and general boundary conditions. - International Journal of Engineering Science, vol.74, pp.1-14.

[20] Morini L., Piccolroaz A. and Mishuris G. (2014): Remarks on the energy release rate for an antiplane moving crack in couple stress elasticity. - International Journal of Solids and Structures, vol.51, No.18, pp.3087-3100.

[21] Şimsek M. (2014): Nonlinear static and free vibration analysis of microbeams based on the nonlinear elastic foundation using modified couple stress theory and He's variational method. - Composite Structures, vol.112, pp.264-272.

[22] Ashoori A. and Mahmoodi M. (2015): The modified version of strain gradient and couple stress theories in general curvilinear coordinates. - European Journal of Mechanics-A/Solids, vol.49, pp.441-454. 
[23] Askari A.R. and Tahani M. (2015): Size-dependent dynamic pull-in analysis of beam-type MEMS under mechanical shock based on the modified couple stress theory. - Applied Mathematical Modelling, vol.39, No.2, pp.934-946.

[24] Dehrouyeh-Semnani A.M. and Nikkhah-Bahrami M. (2015): A discussion on incorporating the Poisson effect in microbeam models based on modified couple stress theory. - International Journal of Engineering Science, vol.86, pp.20-25.

[25] Farokhi H. and Ghayesh M.H. (2015): Nonlinear dynamical behaviour of geometrically imperfect microplates based on modified couple stress theory. - International Journal of Mechanical Sciences, vol.90, pp.133-144.

[26] Tadi Beni Y., Mehralian F. and Razavi H. (2015): Free vibration analysis of size-dependent shear deformable functionally graded cylindrical shell on the basis of modified couple stress theory. - Composite Structures, vol.120, pp.65-78.

[27] Biot M. (1956): Thermoelasticity and irreversible thermodynamics. - Journal of Applied Physics, vol.27, pp.240253.

[28] Lord H. and Shulman Y. (1967): A generalized dynamical theory of elasticity. - Journal of Mechanical Physics, vol.15, pp.299-309.

[29] Green A.E. and Lindsay K.A. (1972): Thermoelasticity. - Journal of Elasticity, vol.2, pp.1-7.

[30] Ram P. and Sharma N. (2008): Reflection and transmission of micropolar thermoelastic waves with an imperfect bonding. - International Journal of Applied Math and Mechanics, vol.4, No.3, pp.1-23.

[31] Xue B.B., Xu H.Y., Fu Z.M. and Sun Q.Y. (2010): Reflection and refraction of longitudinal displacement wave at interface between two micropolar elastic solid. - Advanced Materials Research, vol.139 - 141, pp.214-217.

[32] Kumar R., Kaur M. and Rajvanshi S.C. (2014): Reflection and transmission between two micropolar thermoelastic half-spaces with three-phase-lag model. - Journal of Engineering Physics and Thermophysics, vol.87, No.2, pp.295-307.

[33] Kumar R., Kumar K. and Nautiyal R.C. (2015): Reflection at the free surface of couple stress generalized thermoelstic solid half-space. - Open Journal of Heat, Mass and Momentum Transfer, Accepted.

Received: April 16, 2015

Revised: December 23, 2015 